
entgegenschlägt“ (Kathrin Röggla)

\author{
Klimawandel und Wetterbericht in ausgewählten Werken von \\ Marcel Beyer und Kathrin Röggla
}

\begin{abstract}
Eva Horn prägt den Begriff „Katastrophe ohne Ereignis“ und versteht darunter die nicht enden wollende Kette von Desastern als Folge der Umweltzerstörung, die gerade wegen ihrer Alltäglichkeit der Aufmerksamkeit der Medienrezipienten in der Regel entgeht. Desastern anthropogenen Ursprungs, z.B. schweren Störfällen in technischen Bereichen, kann meist durch Vorsichtsmaßnahmen vorgebeugt werden. In Bezug auf Naturkatastrophen als Folge des Klimawandels ist das nicht mehr der Fall. Der Durchschnittsbürger fühlt sich hier zudem wenig verantwortlich, auch weil sich die katastrophalen Naturphänomene nicht eindeutig als Konsequenzen der industriellen Entwicklung einordnen lassen. Nitzke sieht den Klimawandel daher als „Apokalypse von innen“, als den von der Menschheit selbstverschuldeten Untergang unserer Spezies auf diesem Planeten. Auch die zeitgenössischen deutschsprachigen Autoren Kathrin Röggla und Marcel Beyer sehen die Auswirkungen der Klimakatastrophe als kritisch für die Menschheit. Ausgehend von dem von Nitzke geprägten Terminus weist der vorliegende Beitrag auf die den Autoren jeweils eigenen stilistischen Mittel hin, mit denen Kathrin Röggla und Marcel Beyer mögliche und schon aufgetretene Folgen der „Apokalypse von innen“ erfassen, um die Leser und damit auch deren soziales Umfeld aufzurütteln.
\end{abstract}

Keywords: Marcel Beyer; Kathrin Röggla; Ökokritik; Ecocriticism; Umweltzerstörung; Klimawandel in der Literatur; Wetterbericht; Katastrophe; Apokalypse von innen; Ausnahmezustand; Wasserstandsbericht; Alarmbereitschaft; New Journalism

\title{
1 Einleitung und Zielsetzung
}

Die Naturwissenschaften konzentrieren sich zunehmend auf die Folgen des Klimawandels, die als ernste Gefahr für die Menschheit und unsere Zivilisation gesehen werden. (Vgl. u. a. Randers 2012) Claus Leggewie und Harald Welzer sehen die 
Klimaerwärmung als „die größte Krise der Nachkriegszeit“ und appellieren an die Bürger, ihren Lebensstil zu ändern. (Vgl. Leggewie und Welzer 2011, 20) Die Medien warnen vor Gesundheitsrisiken aufgrund der steigenden Umweltverschmutzung, sie konfrontieren den Nutzer mit furchterregenden Katastrophenbildern, mit denen sie die Auswirkungen des Treibhauseffekts vor Augen führen. Überall trifft der Medienkonsument auf Bilder des Elends und der Zerstörung, fühlt sich dabei jedoch erstaunlich unbeteiligt. Übersättigt und abgestumpft durch sich überbietende Katastrophenberichte verweigert er Interesse und Mitgefühl. (Vgl. Sontag 2003a, 2003b und 2007c; vgl. auch Herring und Sorrell 2009) Als Mitglied einer schnelllebigen konsum- und karriereorientierten Gesellschaft sind Aufnahmefähigkeit und -wille eng getaktet und werden nur durch noch extremere rationale und emotionale Provokationen herausgefordert. So empfindet der Medienkonsument die wiederholten Warnungen der Ökokritiker als bloße Floskeln. Desinteressiert nimmt er die zeitaufwändigen, für ihn ergebnislos scheinenden Debatten der internationalen Konferenzen zum Klimaschutz zur Kenntnis und kann keinen politischen Konsens erkennen. ${ }^{1}$ Solche Debatten, wie auch deren mediales Abbild, sehen politisch engagierte Schriftsteller wie Marcel Beyer und Kathrin Röggla mit viel Skepsis.

Für die Vertreter der Gegenwartsliteratur drängen sich die Fragen auf, mit welchen Mitteln heutige Künstler die aktuelle Klimadebatte beeinflussen können und wie sie diese Diskurse in ihrem Schaffen verarbeiten. ${ }^{2}$ Die Antworten von Beyer und Röggla werden im Folgenden an ausgewählten Werken dieser Schriftsteller untersucht, unter Berücksichtigung des von Solvejg Nitzke geprägten Begriffs der „Apokalypse von innen“ (Nitzke 2012, 176), hauptsächlich anhand der Texte aus dem Prosaband Nonfiction des deutschen Schriftstellers (Beyer 2003) und dem Prosaband die alarmbereiten der österreichischen Autorin (Röggla 2010a), in denen Naturdesaster infolge des Klimawandels einen wesentlichen Platz einnehmen.

Während im Alten Testament ungewöhnliche Naturphänomene als Strafe Gottes und Vorzeichen der vorhergesagten Apokalypse gelten, bemühen sich einige Schriftsteller der Epoche der Aufklärung, u.a. Voltaire in seiner Deutung

1 Dieses Problem ist Gegenstand von Kathrin Rögglas Theatertext die unvermeidlichen (Mannheim 2011).

2 Zum Diskurs-Begriff vgl. u. a. Burtscher-Bechter 2004. Link und Link-Heer unterscheiden zudem zwischen dem „Spezialdiskurs“ und dem „Interdiskurs“. (Link und Link-Heer 1990) Die Interdiskursanalyse untersucht „kulturell vermittelte und institutionalisierte Formen der Rede in verschiedenen gesellschaftlichen Praxisbereichen sowie deren Beziehungen untereinander“. (Link und Parr 2005, 108) Das Fachwissen werde aus den Spezialdiskursen in den medialen Interdiskurs aufgenommen. 
des Erdbebens von Lissabon, um eine andere Interpretation von Naturdesastern. (Vgl. Voltaire 1912 und 1994) Aufgrund von naturwissenschaftlichen Erkenntnissen ihrer Zeit sehen sie überraschende gewaltige Wetterphänomene als weder vom Menschen noch von der Vorsehung abhängige Ereignisse. Laut François Walter unterscheiden die meisten Katastrophenforscher zwischen „dem irrationalen, für die alten Gesellschaften charakteristischen Umgang mit Unglücksfällen“ und der „wissenschaftlichen Bewältigung, wie sie modernen Gesellschaften eigen sind“. (Walter 2008, 10) Der Autor stellt fest: „Religiöse Deutungsmuster hätten sich in der ländlichen Bevölkerung teilweise bis ins 20. Jahrhundert hinein gehalten, während sich die städtischen Eliten seit dem 18. Jahrhundert dem neuen, naturwissenschaftlichen Weltbild zuwandten.“(Walter 2008, 13) Walter betont zudem, dass „religiöse und symbolische Erklärungsschemata global und langlebig sind und dass ihr Wirkungsfeld weit über das Zeitalter der sogenannten Aufklärung hinausreicht, in dem die Desakralisierung der Welt den Geltungshorizont dieses Denkmusters endgültig in die Vergangenheit zu verweisen schien.“ (Walter 2008, 12)

Katastrophen infolge von Umweltzerstörung zeigen selten ausschließlich lokale Wirkung und müssen als globales Phänomen gesehen werden. ${ }^{3}$ Die Menschheit ist in ihrer Existenz gefährdet, da die Spätfolgen der ungezügelten Zivilisation den Lebensraum zerstören - ein Gedanke, den u.a. Günther Anders formuliert. (Vgl. Anders 1956 und 1980) Im Gegensatz zu den in der Bibel prophezeiten Plagen ist der Mensch für die langfristigen Folgen der Umweltzerstörung mitverantwortlich.

Wissenschaftler und Schriftsteller sehen den Klimawandel heutzutage als unabwendbar an; Ausmaß und Folgen könnten nur abgeschwächt werden. ${ }^{4}$ Im Fall der Desaster, die als Folgen des Klimawandels auftreten, lassen sich, so Solvejg Nitzke, die natürlichen und die von Menschen ausgelösten Ursachen nicht zuordnen. (Vgl. Nitzke 2012, 176) Die Literaturwissenschaftlerin spricht daher von der realen „Bedrohung von innen“, die die bestehende Ordnung zersetzt und sich wie eine Seuche ausbreitet. (Nitzke 2012, 176) Im Gegensatz zu einigen anderen großen Unglücksfällen, wie z. B. schweren Betriebsunfällen, seien Naturkatastrophen als Folge des Klimawandels nicht zu verhindern. Für diese unausweichlichen Entwicklungen erscheint der Apokalypsen-Begriff zutreffend.

3 Nur auf wenige Weltgegenden beschränkt sind positive Folgen des Klimawandels zu verzeichnen, wie z. B. die Veränderungen für die Vegetation in kälteren Erdteilen.

4 In ihrem Beitrag „Das große Unsichtbare. Die Modellierung von Klima zwischen Wissenschaft und Literatur" verweist Nitzke darauf, dass das Klima heute ein wandelbares globales Phänomen ist, dessen Auswirkungen erst im Ansatz erkennbar sind. (Nitzke 2016, 90) 
Im 21. Jahrhundert bemühen sich einige ökokritische Autoren um eine realitätsnahe Darstellung gegenwärtiger Anzeichen von Klimawandel. ${ }^{5}$ Die Hauptfigur in Ilja Trojanows Roman Der EisTau (2011), ein Glaziologe namens Zeno, untersucht die Auswirkungen der globalen Erwärmung auf die Natur, eine Entwicklung, die u.a. auch von Kathrin Röggla im Erzählband die alarmbereiten (1995) aufgegriffen wird. Hier benutzt der Schriftsteller eine Schiffbruchmetapher, um auf den möglichen Untergang unserer Zivilisation hinzuweisen. Im Zusammenhang mit der steigenden Ökonomisierung aller Lebensbereiche beurteilen viele Autoren zudem kritisch den Umstand, dass in der New Economy der Klimawandel als Anlass für neue Geschäftsmodelle dient. Neben dem Roman EisTau kann hier als Beispiel Ian McEwans Werk Solar (2010) genannt werden, wo ein PhysikNobelpreisträger namens Michael Beard geschäftstüchtig von der Angst vor der globalen Erwärmung profitiert. Die Konfrontation der Ideale eines Umweltschützers mit einer harten, durch ökonomische Kriterien geprägten Realität präsentiert Jonathan Franzen in seinem Bestseller Freedom (2011). Hier stellt der Autor einen Umweltaktivisten im Kampf für eine bedrohte Art von Singvögeln vor. Dieses lobenswerte Ziel steht jedoch in Konflikt mit pragmatischen und persönlichen Interessen des Protagonisten.

Die Symptome des Umweltdesasters, von denen die Medien immer wieder berichten, erregen selten die Aufmerksamkeit der Fernsehzuschauer. Nur die besonders sensiblen Menschen befürchten, dass sich diese Hinweise auf die globale Deregulierung der komplexen Systeme erhärten und letztendlich ein Punkt erreicht wird, von dem es kein Zurück mehr gibt: der Stillstand der Gezeiten und der Untergang der uns vertrauten Welt.

Einige Wissenschaftler betonen, dass Menschen Katastrophen, insbesondere Naturdesaster, erst dann wahrnehmen, wenn sie von ihren Folgen unmittelbar betroffen sind. In Zukunft als Katastrophe (2014) stellt Eva Horn fest: „Nicht zufällig sind die Katastrophenszenarien, die heute am intensivsten diskutiert werden [...], die Deregulierungen komplexer Systeme“. (Horn 2014, 19) Sie versteht darunter die Zerstörung des Gleichgewichts innerhalb der Ökosysteme (z. B. in der Unterwasserwelt) als Folge des weltweiten Klimawandels. ${ }^{6}$ Die Folgen der Umweltzerstörung werden von den heutigen Menschen jedoch nicht zur Kenntnis genommen, auch wenn sie über ein gewisses Maß an Grundwissen um den Zustand der Naturwelt verfügen. Eva Horn betont daher, wir lebten „in einer Gegenwart, die von höchst diffusen Zukunftsszenarien und einer drohenden Katastrophe ohne Ereignis geprägt ist“. (Horn 2014, 19) Bedrohliche Wetter-

5 Eine Auswahl von ökokritischen Prosatexten und Gedichten bietet auch der Band „unter sterbenden bäumen“. Grüne Literaturgeschichte. (Vgl. Krüger 2001)

6 Zum Klima-Begriff vgl. u. a. Horn 2017, Kelley 2015 und Nitzke 2016. 
erscheinungen erweisen sich heutzutage nicht als einzelne Störfälle (um Christa Wolfs Worte zu verwenden [vgl. dazu Wojno-Owczarska 2016]), sondern als Teile eines bereits in Gang gesetzten Prozesses. Diese schleichenden Veränderungen, deren endlose Kette vom Ausmaß der Umweltzerstörung zeugt, werden von Eva Horn als eine „Katastrophe ohne Ereignis“ charakterisiert. (Horn 2014, 19)

Das immerwährende Gefühl von Bedrohung lässt auch literarische Bilder entstehen, die eine Apokalypse als Folge von Mangel an Trinkwasser und Nahrungsmitteln und in Form von Hitzewellen oder gewaltigen Regenfällen heraufbeschwören. Als Ausgangspunkt der weltweiten Umweltbewegung wird Rachel Carsons Roman Silent Spring (1962) gesehen. (Vgl. Carson 1962) Auch in der Sciencefictionliteratur stellen Autoren weltweit apokalyptische Szenarien bedingt durch die Naturkatastrophen dar und verweisen auf das Fehlverhalten des Einzelnen angesichts einer durch Extremwetterereignisse bedingten Krise; so z. B. Octavia Butlers The Parable of the Sower (1993) und Thomas Coraghessan Boyles A Friend of the Earth (2000). Um weitere literarische Beispiele zu nennen: In Mara and Dann: An Adventure (1999) zeigt die Nobelpreisträgerin Doris Lessing eine Welt, in der die Menschen dem Wechsel von Dürre und Überschwemmungen ausgesetzt sind. Cormac McCarthy schildert in seinem Roman The Road (2006) ${ }^{7}$ das Schicksal von wenigen Überlebenden, die wegen der zunehmenden Umweltverschmutzung um Nahrung und Trinkwasser kämpfen. In den erwähnten Werken bringen kaum zu ertragende Lebensbedingungen die Wahrheit über die Charaktereigenschaften der Protagonisten ans Tageslicht.

Der Super-GAU von Tschernobyl (1986) machte den Menschen bewusst, wie verletzlich unsere Existenz auf Erden ist. Auch die Fukushima-Katastrophe (2011) steigerte das Gefühl der Bedrohung. Das Beispiel Fukushima steht für die tragische Verknüpfung der Auswirkung eines Naturdesasters - eines Tsunami - und eines dadurch ausgelösten Reaktorunfalls, der durch unzureichende Vorsichtsmaßnahmen befördert wurde: „Es zeigt also die Unberechenbarkeit und Kraft, letztlich die Dominanz der Natur über den Menschen“. (Hoffstadt 2012, 31) 2011 sehen sich viele Künstler erneut mit der Tatsache konfrontiert, dass ein technischer Defekt einen unabwendbaren „Stillstand der Gezeiten der Natur“ nach sich ziehen kann. (Horn 2014, 165) Sie verweisen auf die Bedrohung der Existenz auf unserem Planeten durch den technischen Fortschritt und durch beängstigende Entwicklungen in der Wissenschaft. Sie bedienen sich erschreckender Bilder, indem sie apokalyptische Szenarien eines nahenden Weltuntergangs skizzieren; eine Maßnahme, die die „Katastrophenlust“ der Kunstrezipienten befriedigen

7 Verfilmt 2009, Regie: John Hillcoat, mit Charlize Theron und Viggo Mortensen in den Hauptrollen. 
soll, um die Aufmerksamkeit der Zuschauer in einer Gesellschaft des Spektakels zu erregen. (Röggla 2005, 31; vgl. Sontag 2003a) Zusammenfassend lässt sich folgern, dass Marcel Beyers und Kathrin Rögglas Sorge um die Umwelt in der Literatur des neuen Jahrtausends nicht allein steht.

Der vorliegende Artikel geht der These nach, dass sich beide Autoren in ihren Prosatexten mit der Wirkung öffentlicher Diskurse über den Klimawandel beschäftigen, die die Debatten im neuen Jahrtausend immer noch prägen. Zudem stellen Marcel Beyer und Kathrin Röggla in ihren Texten den Klimawandel als „Apokalypse von innen“ (Nitzke 2012, 176) dar.

Das Hauptaugenmerk richtet sich im vorliegenden Artikel auf die Frage, inwieweit beide Autoren in ihrem Schaffen Elemente der öffentlichen Klimadiskurse im Original aufnehmen und künstlerisch verarbeiten. Analysiert werden ausgewählte Prosatexte von Kathrin Röggla (die alarmbereiten, Röggla 2010a) und Marcel Beyer (Nonfiction, Beyer 2003), in denen die Umweltproblematik in den Vordergrund tritt. Das fehlende Interesse der schnelllebigen Gesellschaft an Fragen des Umweltschutzes nehmen die Schriftsteller zum Anlass, ihre Kritik an der voyeuristischen Gesellschaft zu äußern. Statt einer dokumentarischen Aufzeichnung von Fakten, die den globalen Temperaturanstieg belegen, berücksichtigen sie die jeweils individuelle Perspektive der Protagonisten und untersuchen den Einfluss medialer Berichte auf den Einzelnen.

\section{Zum Begriff „Ökokritik“}

Wissenschaftler unterschiedlicher Fachrichtungen beschäftigen sich im 21. Jahrhundert mit dem Klimawandel, u. a. der Arzt Ronald D. Gerste und die Historiker Wolfgang Behringer und Frank Uekötter. (Vgl. Behringer 2011; Uekötter 2011; Gerste 2016) Die Auseinandersetzung mit dem Terminus „Ecocriticism“ findet ihren Widerhall auch in der Literaturforschung. Der aus der US-amerikanischen Literaturwissenschaft stammende Begriff „Ecocriticism“ (bzw. „Ökokritik“), der von William Rueckert in seinem Aufsatz Literature and Ecology. An Experiment in Ecocriticism geprägt wurde, hat sich mittlerweile als interdisziplinäre Forschungsperspektive weltweit etabliert. (Vgl. u. a. Rueckert 1978; Ermisch et al. 2010, 3; Glotfelty 1996, XIX; Metzler 2013, 155-157) Als Meilenstein bei der Entstehung der Ökokritik wird zudem das Panel Ecocriticism: The Greening of Literary Studies genannt, das bei der Jahrestagung der Modern Language Association 1991 abgehalten wurde. (Vgl. Starre 2010, 16) 1993 wird von Patrick Murphy die Zeitschrift Interdisciplinary Studies in Literature and Environment gegründet. Auch im deutschsprachigen Raum zeichnet sich ein wachsendes Interesse an ökokritischen Studien und 
Werken ab. ${ }^{8}$ Um eine kritische Aufbereitung und Weiterentwicklung der angloamerikanischen Ansätze bemühten sich u. a. Zapf (2002), Grewe-Vollp (2004) und Hofer (2007). Aufgrund der zunehmenden Internationalisierung wird von einigen Literaturwissenschaftlern eine komparatistische Perspektive auf die Umweltorientierung der unterschiedlichen nationalen Literaturen gefordert. (Vgl. Murphy 2000) Als Beispiel kann die von Gabriele Dürbeck geleitete Sektion Klimachaos und Naturkatastrophen in der deutschen Literatur - Desaster und deren Deutung während der XII. IVG-Tagung in Warschau gelten. ${ }^{9}$ (Vgl. Grucza et al. 2012)

Der Terminus „Ökokritik“ spielt auch die zentrale Rolle in dem von Gabriele Dürbeck und Urte Stobbe edierten Band Ecocriticism. Eine Einführung (2015). Einen wichtigen Platz in den gegenwärtigen Klimadiskursen nehmen zudem die Arbeiten der Amerikanistin Evi Zemanek und der Germanistinnen Eva Horn und Solvejg Nitzke ein. (Vgl. u. a. Zemanek 2011, Horn 2011, 2014 und 2017; Nitzke 2012, 2016 und 2017; Nitzke und Pethes 2018) Als Beispiel für aktuelle ökokritische Studien sei auch der von Eva Horn und Peter Schnyder herausgegebene Band Romantische Klimatologie genannt, der Texte zum modernen Verständnis des Klima-Begriffs enthält. (Vgl. Horn und Schnyder 2016)

Vom 21. bis 27. Juli 2016 tagte in Wien die Internationale Vereinigung der Komparatisten, deren Beiträge sich auf den globalen Klimawandel konzentrierten. Im Zusammenhang mit den literarischen Klimadiskursen sind insbesondere die Beiträge des von Eva Horn und Solvejg Nitzke geleiteten Panels Languages of Climate - Comparative Approaches to the Environment $\mathrm{zu}$ beachten, $\mathrm{u}$. a. die von Benjamin Bühler, Susanne Fuchs und Patrick Stoffel, die sich in ihren Arbeiten mit der Geschichte des Klima-Begriffs und mit Extremwetterereignissen als Folgen des globalen Klimawandels beschäftigen. Zudem leitete Gabriele Dürbeck eine Sektion zu literarischen Darstellungen des „Anthropozän“, ${ }^{10}$ eines kontrovers diskutierten Begriffs, der immer häufiger im Zusammenhang mit literarischen Klima-

81970 erscheint die erste Ausgabe der Zeitschrift Umwelt, ein Jahr später Das technische Umweltmagazin. Seit den 1980er Jahren nehmen die Grünen an öffentlichen Debatten teil.

9 Aufgrund des beschränkten Umfangs der vorliegenden Studie wird hier nur ansatzweise auf Beispiele von Arbeiten deutschsprachiger Forscher hingewiesen, die sich mit den literarischen Motiven des Klimawandels und der Umweltzerstörung beschäftigen, insbesondere derjenigen Literaturwissenschaftler, die sich auch an der ICLA-Tagung in Wien 2016 beteiligten. Zum Zeitpunkt der Einreichung des vorliegenden Beitrags (2016) sind alle Bände bezüglich der ICLA-Tagung noch in Vorbereitung.

10 Dieser Terminus wurde 2002 vom Nobelpreisträger für Chemie Paul Crutzen in einem NatureArtikel geprägt. (Vgl. Crutzen 2002) Bereits am Ende des 18. Jahrhunderts habe im Zusammenhang mit dem Einbruch der Industrialisierung ein neues Zeitalter begonnen. Charakteristisch für die neue Ära seien die Veränderung der Umwelt, der Anstieg der Treibgaswerte, die Nutzung von 30-50 \% der Landoberfläche durch den Menschen und die Ausbeutung der Meere. 
diskursen fällt. (Vgl. u. a. Dürbeck 2001, 2007, 2010, 2012 und 2017; Kersten 2014 und Wilke 2014) Zu den aktuellen ökokritischen Studien gehören auch die 2017 erschienenen Publikationen Climate Change, Complexity, Representation (Hg. Hannes Bergthaller und Arndt Niebisch), German Ecocriticism in the Anthropocene (Hg. Caroline Schaumann und Heather I. Sullivan), Imagining Earth. Concepts of Wholeness in Cultural Constructions of „Our Home Planet“(Hg. Solvejg Nitzke und Nicolas Pethes), Literatur und Ökologie. Neue literatur- und kulturwissenschaftliche Perspektiven (Hg. Christiane Solte-Gresser und Claudia Schmitt) und Meteorologies of Modernity: Weather and Climate Discourses in the Anthropocene (Hg. Sarah Fekadu, Tobias Döring und Hanna Straß-Senol). Empfehlenswert ist zudem der von Dürbeck, Kanz und Zschachlitz herausgegebene Band Ökologischer Wandel in der deutschsprachigen Literatur des 20. und 21. Jahrhunderts: Neue Perspektiven und Ansätze (2017). Die Bände Topografische Leerstellen - Ästhetisierungen verschwindender und verschwundener Dörfer und Landschaften in Literaturen, Filmen und Künsten (Hg. Marc Weiland und Martin Ehrler) und Narrative Strategien des Anthropozän in deutsch- und englischsprachiger Literatur und in den Medien/ Narratives of the Anthropocene in German- and English-Speaking Literature and in the Media (Hg. Gabriele Dürbeck und Jonas Nesselhauf) sind zum Zeitpunkt der Einreichung des vorliegenden Beitrags in Vorbereitung. Die Anzahl der kürzlich entstandenen Publikationen zu ökokritischen Texten zeugt von der Aktualität der Forschung über die Darstellung des Klimawandels in der Literatur.

\section{Die Darstellung des Klimawandels in Kathrin Rögglas Werk}

Nach Rögglas eigener Aussage haben die Texte der deutschen Vertreter der Ökokritik ihre Arbeit weniger beeinflusst als die Darstellung von Katastrophen in Mike Davis' Ökologie der Angst (Ecology of Fear, 1998) oder Günther Anders' Zivilisationskritik im Werk Die Antiquiertheit des Menschen. ${ }^{11}$ Im Prosaband die alarmbereiten (1995) der österreichischen Autorin verfolgt eine junge Frau mit dem vielsagenden Namen Kassandra Nachrichten über Waldbrände, die an Davis' Darstellung von Katastrophen in Kalifornien erinnern. (Vgl. Röggla 2010a, 33) In Rögglas Erzählband Nachtsendung. Unheimliche Geschichten fragt der Erzähler explizit, ob heute nur noch eine „Welt ohne Menschen“ vorstellbar sei, und bezieht sich dabei auf den Titel von Günther Anders’ Werk Mensch ohne Welt. (Röggla 2016, 7; vgl. Anders 1984)

11 Eigenes, bisher nicht veröffentlichtes Interview mit der Autorin (2012). 
Im Erzählband die alarmbereiten warnt die Autorin vor den negativen Folgen des Klimawandels, die bereits Realität geworden sind. (Vgl. Röggla 2010a) Anders als etwa in Ilja Trojanows EisTau sind hier jedoch nicht die Folgen des Klimawandels für die Naturwelt das Hauptthema, sondern die Wirkung medialer Diskurse auf den Einzelnen: „Der Konjunktiv und die Verdopplung der Sprechsituation ermöglichen es, die medial und institutionell gesteuerten Diskurse und die sozialen Dispositive offenzulegen.“ (Rutka 2014, 102; vgl. Trojanow 2011)

Die empfindsame Kassandra in Rögglas Erzählband sammelt anhand medialer Berichte Hinweise auf Symptome der ökologischen Katastrophe. Nachts verfolgt sie Radionachrichten über die Erhöhung der Wassertemperatur, den steigenden Salzgehalt der Meere und die Folgen für das Plankton. Im Internet sucht sie nach Fotos von bereits eingetretenen Zerstörungen, z. B. der als Folge der Dürre „abgestorbenen bäume und baumstümpfe“. (Röggla 2010a, 35) Die medialen Berichte über den Treibhauseffekt lösen bei der jungen Frau große Angst aus, so dass sie mitten in der Nacht über das Telefon nach menschlicher Zuwendung sucht. Nach Rögglas eigener Aussage ${ }^{12}$ sollten die alarmbereiten ursprünglich ein umgekehrter Entwicklungsroman werden. Statt ihr Wissen über die Welt zu erweitern, verliert Kassandra den Überblick über die durch eigene Nachforschungen gewonnenen Informationen und verwandelt sich in ein „recherchegespenst“, ein furchterregendes „phantom“; das sprechende Subjekt tritt hinter die vermittelten Informationen zurück. (Röggla 2010a, 93) Am Beispiel der sensiblen Frau wird in Rögglas Werk veranschaulicht, dass die öffentlichen Klimadiskurse für sogenannte Durchschnittsbürger kaum nachvollziehbar sind; auch ist keine Lösung der anstehenden Probleme abzusehen. Laut Kassandra führt der Klimawandel zu einer endgültigen Vernichtung der Gattung Mensch; andere Plagen von heute, wie $u$.a. der Rinderwahnsinn oder die Vogelgrippe, seien nur erste Symptome dieser „Apokalypse von innen“, „die der klimageschichte vorausgegangen seien“. (Nitzke 2012, 176; Röggla 2010a, 38) Dieser Aspekt des Werks fand in der RögglaForschung bisher wenig Beachtung, obwohl er in ihrem Text explizit betont wird. ${ }^{13}$

Die österreichische Autorin führt in ihrem Erzählband dem Leser vor Augen, dass die komplexen Prozesse, die mit der Umweltzerstörung verbunden sind, die Vorstellungskraft der meisten Medienrezipienten übersteigen. Die Realität entzieht sich einer naturalistischen Darstellung, und das umso mehr, als der Einzelne nur ihr mediales Abbild erfährt. Die Symptome des Klimawandels werden in Rögglas Erzählband von einer empfindsamen Frau namens Kassandra auf-

12 Eigenes, bisher nicht veröffentlichtes Interview mit der Autorin (2012).

13 Die Dissertation Seit Jahrzehnten „fünf nach zwölf“ von Mara Stuhlfauth-Trabert ist zum Zeitpunkt der Einreichung des vorliegenden Beitrags in Vorbereitung. (Vgl. Stuhlfauth-Trabert 2017) 
gezeichnet: „sie wolle eher abschalten. aber man dürfe nicht abschalten, habe sie recht?“. (Röggla 2010a, 30) Die Fachsprache der Meteorologie und die Fülle an Informationen überfordern jedoch Kassandras Aufnahmefähigkeit: ,ich hätte begriffe wie ,el niño' oder ,nordatlantische oszillation‘ ebenso wenig alleine gepachtet wie ich die alleineigentümerin von klimadaten sei.“ (Röggla 2010a, 32) Die junge Frau scheitert jedoch an dem Versuch, diese Phänomene zu systematisieren und einen kausalen Zusammenhang nachzuweisen.

In Rögglas stark durch Ökokritik geprägtem Werk ist Kassandra als Seherin in unserer Zeit die Einzige, die Berichte über den Klimawandel mit der Gefahr der totalen Auslöschung des Lebens assoziiert; dieser Zukunftspessimismus wird ihr allerdings von ihren Mitbürgern vorgeworfen: „bei mir denke man bei einem begriff wie ,polardrift" automatisch an einen untergang, an ein endzeitgeschehen. “ (Röggla 2010a, 32) Die Figur wirkt von der Gesellschaft isoliert. Ihren Worten schenkt man keinen Glauben, wie es einst der mythischen Kassandra-Figur erging: „ich mit meinen klimakatastrophen, die mir nicht auszureichen scheinen, ich mit meinen umweltgiften, ja, ich mit meinen wetterereignissen und mit meinen krankheitserregern, die ich in einen zusammenhang bringen würde“. (Röggla 2010a, 38) Auch von ihrer Gesprächspartnerin wird sie abgelehnt: „der ständige alarm habe zur folge, dass mir niemand mehr zuhören wolle.“ (Röggla 2010a, 39)

Der Anfang des ersten Textes im Erzählband die alarmbereiten steigt in die Beschreibung imaginärer Katastrophen ein, die der Menschheit drohen, und liefert Hinweise bezüglich der Interpretation des Werks. Es geht hier weniger um die Darstellung einer von den Folgen des Klimawandels geprägten Realität als um die Wirkung medial vermittelter Diskurse. Mit einer fast gleichlautenden Einleitung beginnt auch das Theaterstück worst case (UA Freiburg 2008), Rögglas Adaptation des Erzählbandes die alarmbereiten für die Bühne:

mal sehen, ob die wälder wieder brennen, mal sehen, ob eine hitze uns entgegenschlägt. mal sehen, ob der rauch die tiere aus den büschen treibt, deren namen wir nicht kennen, mal sehen, ob das eine stille nach sich zieht. mal sehen, ob der regen einsetzt, den ein schwarzer wind ins land drückt, mal sehen, ob sich wassermassen gegen brücken stemmen oder dämme längst gebrochen sind.

mal sehen, ob gebäudeteile auf uns zukommen, ja, mal sehen, ob das ganze runterkommt und eine staubwolke uns entgegenschlägt, die alle farben schluckt. mal sehen, ob eine stromleitung auf der fahrbahn liegt. ${ }^{14}$ (Röggla 2008, 7; vgl. Röggla 2010a, 2)

142008 wurde Rögglas Hörspiel das recherchegespenst ausgestrahlt (BR 2008). Für den Theatertext worst case (UA Freiburg, 2008) bekam die Schriftstellerin 2010 den Wiener Theaterpreis „Nestroy“ als „Beste Autorin“. 2009 wurde zudem Rögglas Hörspiel die alarmbereiten vom Bayerischen Rundfunk gesendet. 
Die Sprachsymbolik hat in diesem Fragment viel gemein mit der Darstellung von Plagen alttestamentarischen Ausmaßes und biblischen Prophezeiungen über den Einbruch der Apokalypse: Eine weiträumige Überschwemmung gemahnt an die Sintflut, die nur Gerechte überleben; der von einer „staubwolke“ gefolgte „schwarze wind“ erinnert an die sieben Plagen Ägyptens (2 Mose 7, Exodus) und die Weissagungen der Offenbarung (Offb. 15,16). (Röggla 2008, 2; vgl. Röggla 2010a, 7) Der schwarze Wind kann jedoch auch als ein Hinweis auf den Grad der Luftverschmutzung oder auf die Folgen eines Super-GAUs gesehen werden.

Die durch mediale Bilder zahlreicher Naturdesaster beunruhigte Kassandra lebt in Erwartung neuer Nachrichten von gewaltigen Regenfällen, dem Einbruch einer neuen Eiszeit, riesigen Bränden, vielleicht auch atomaren Explosionen mit darauf folgenden zerstörerischen Winden als Konsequenzen aus dem vom Menschen zu verantwortenden Frevel an der Natur. (Vgl. Röggla 2010a, 7 und 31) Daher hat die These Bestand, dass die Autorin in ihrem Erzählband den Klimawandel als „Apokalypse von innen“ darstellt, deren natürliche und anthropogene Ursachen nicht mehr trennbar sind. Röggla benutzt zwar bildhafte Mittel, die den Lesern Kassandras fragile Existenz in einer Welt ständiger Bedrohung bewusst machen, jedoch geht es ihr hier vor allem um die Wirkung medialer Diskurse im Bereich des Klimawandels. Der heutige Mensch wird von den Medien mit einem Übermaß an Nachrichten überhäuft, so dass er Unwetterwarnungen und Berichte über weitere Folgen des Klimawandels nicht zur Kenntnis nimmt: „mal sehen, ob sie wieder auf der brücke stehen und hinuntersehen, einen steinwurf weg von ereignissen, die sie doch nicht verstehen. mal sehen, ob sie dann zu anderen dingen übergehen, weil ihnen gar zu langweilig wird. mal sehen, ob sich wieder was tut.“ (Röggla 2010a, 7)

Die zitierte Textpassage lässt an aufeinander folgende mediale Katastrophenszenen denken. Anhand medialer Berichte verfolgt Kassandra die Zeichen der fortschreitenden ökologischen Katastrophe als einer durch den Menschen verursachten „Bedrohung von innen“. (Nitzke 2012, 176) Sie erfährt nur ein mediales Abbild der Realität, da sie sich immer mehr in ihr abgeschottetes Zuhause zurückzieht. Rögglas Werk veranschaulicht, dass nur sensible Menschen wie Kassandra eine solche Beunruhigung durch mögliche Untergangsszenarien infolge des globalen Temperaturanstiegs verspüren. Die Vorstellungskraft der jungen Frau ist dermaßen von medial vermittelten Prognosen determiniert, dass sie sich die schlimmsten Szenarien des nahenden Untergangs erdenkt und das Eintreten von spektakulären Wettererscheinungen erwartet, die auch in diesem Textabschnitt an apokalyptische Visionen erinnern: „wo sind sie, die hereinstürzenden fluten, die unter dem druck des sturms einfach zusammenbrechen?““ (Röggla 2010a, 35) Die erwarteten „massenmigrationen“ verschiedener Tierarten infolge des Klimawandels, die z. B. der Glaziologe Zeno in Ilja Trojanows EisTau (2011) und der Biologe Sigur Johanson in Frank Schätzings Der Schwarm (2004) am Beispiel 
der Meeresfauna beobachten, bleiben in Rögglas Erzählband zwar aus; jedoch ist Kassandra sicher, dass die ersten Anzeichen der „Apokalypse von innen“, die zur selbstverschuldeten Ausrottung der Gattung Mensch führt, bereits eingetreten sind. (Röggla 2010a, 32; Nitzke 2012, 176; vgl. Horn 2014, 35) Erkennbar sind in Rögglas Werk auch Bezüge zu Naomi Kleins Schriften über den KatastrophenKapitalismus (vgl. u. a. Klein 2007a und 2007b), die die österreichische Autorin rezipiert: Eine Gesellschaft, die um die Sicherung ihrer existenziellen Bedürfnisse bangen muss, stellt weniger Ansprüche auf Selbstbestimmung und ist leichter beherrschbar. (Vgl. auch Wuketis, 2012) In diesem Kontext kann man katastrophische Visionen der Auslöschung der Menschheit infolge von Dürren, Flächenbränden, atomaren Explosionen oder heftigen Regenfällen in Rögglas Werk deuten.

In ihrem Erzählband zeigt Röggla, dass der Mensch unter dem Einfluss medialer Klimadiskurse vor allem fürchtet, in schwierigen Zeiten nur auf sich allein gestellt zu sein, was auf ihn noch bedrohlicher wirkt als eine reale, immanente Katastrophe. ${ }^{15}$ Diese Angst zeigt sich u. a. in den Worten einer namenlosen männlichen Figur im Text die zuseher: ,wir beide sollten doch nur einmal hinhören: alleine, wie die möwen schrien, alleine, wie der wind das gras bewege. man nehme ja nur noch die lastwagen in der ferne wahr, wie sie vorüberzögen, dabei könne es gar keine lastwagen mehr geben, die vorüberzögen. es sei niemand mehr da. da sei nur das rauschen des windes“. (Röggla 2010a, 25) Die Autorin stellt hier die letzten Menschen dar, die noch ihr Leben auf der Erde fristen, eine literarische Vision, die für moderne Endzeit-Vorstellungen charakteristisch ist und deren Auftreten in Literatur, Film und Malerei u. a. Eva Horn in ihrem Buch Zukunft als Katastrophe (2014) ausführlich analysiert. (Vgl. Horn 2014; vgl. auch Schossböck 2012) Statt einer realitätsnahen Wirklichkeitsdarstellung werden im Erzählband die alarmbereiten mögliche Folgen des Klimawandels in Betracht gezogen: eine Vision der „Apokalypse von innen“, deren Ursachen sich der Vorstellungskraft eines Laien entziehen, deren Eintreten jedoch allem Anschein nach zu erwarten ist. (Nitzke 2012, 176)

\section{Das Verhältnis von Fakt und Fiktion und der Gebrauch der meteorologischen Fachsprache}

Marcel Beyer und Kathrin Röggla thematisieren in ihren Texten das Verhältnis von Fakt und Fiktion und greifen die Frage nach den Beziehungen zwischen Literatur

15 Vgl. u.a. den Text die zuseher. (Röggla 2010a, 29) 
und Journalismus auf, wobei sie auf die fortschreitenden Umweltbelastungen als eine „Apokalypse von innen“ verweisen. (Vgl. Nitzke 2012, 176) Beide Künstler sind Augenzeugen von realen, medial vermittelten Katastrophen, Kathrin Röggla als Stipendiatin des Deutschen Literaturhauses in New York, ${ }^{16}$ Marcel Beyer als Einwohner von Dresden. Für Beyer und Röggla ist auch intensive Recherchearbeit charakteristisch, deren Ergebnisse sie in ihren Texten nutzen. In ihren Werken really ground zero (Röggla 2001) und „Wasserstandsbericht“ (Beyer 2003) konzentrieren sie sich jedoch auf „Erlebnisse Einzelner, sie partikularisieren das Ereignis, um einen identifikatorischen Effekt beim Leser oder Zuschauer zu erreichen“ (Jabłkowska 2012, 35), was sie mit den Vertretern des New Journalism verbindet.

Laut Bleicher erweitern viele Gegenwartsautoren „den Authentizitätsanspruch ihrer fiktionalen Weltentwürfe, indem sie journalistische Darstellungsformen in die fiktionale Weltkonstruktion integrieren [...]. Diese Form der [Einbeziehung] realer Nachrichten [...] lässt die erzählte Fiktion authentischer erscheinen. Die erfundene Handlung wird in einen für den Leser erkennbaren zeithistorischen Kontext eingebunden.“ (Bleicher 2004, 30-31) Dieter Roß spricht in diesem Kontext von der „Journalisierung der Literatur“. (Roß 2004, 77) Der sogenannte New Journalism erweise sich „nicht als eine Programmatik von Journalisten, sondern als ein interessantes Phänomen der wechselseitigen Beeinflussung literarischer und journalistischer Schreibweisen.“ (Bleicher 2004, 29) Beyers „Wasserstandsbericht“ ist ein gutes Beispiel für die o.g. „Journalisierung der Literatur“: Die Geschichte wird in diesem Text aus dem Blickwinkel eines Augenzeugen des Elbhochwassers von 2003 erzählt, wobei auch Fragmente authentischer Wetterberichte in die Erzählung eingewoben werden. Im Gegensatz zu Beyers Prosa stellt der erste Text im Erzählband die alarmbereiten nur potenzielle Fälle von Naturkatastrophen als Beispiele für den fortschreitenden Prozess der „Apokalypse von innen“ vor, er dokumentiert also kein reales Geschehen. Kassandra, die ihr Wissen über die Welt aus den Medien und nicht aus realen Erlebnissen bezieht, wird durch den Mangel an Nachvollziehbarkeit der Inhalte umso stärker beunruhigt und versucht eine eigene Interpretation der medial vermittelten Berichte: „[sie] habe aber die nerven nicht verloren, sie habe sich einen teil dieses vokabulars angeeignet und es sozusagen entschärft“. (Röggla 2010a,

162001 wohnte Kathrin Röggla als Stipendiatin des Deutschen Literaturhauses in New York. Ihr Roman really ground zero basiert auf ihren Erlebnissen als Augenzeugin der Attentate des 11. September 2001. Das Werk erschien zunächst in Abschnitten in der deutschen und der österreichischen Presse (vornehmlich in der Berliner tageszeitung; einzelne Fragmente wurden auch in den Zeitungen Falter und Der Tagesspiegel veröffentlicht). 2002 wurde auch das Hörspiel really ground zero - anweisungen zum 11. september vom Bayerischen Rundfunk ausgestrahlt (Regie: Ulrich Lampen). 
32) Der meteorologische Fachwortschatz wird in Rögglas Erzählband - in abgewandelter Form - in die Passagen eingewoben, in denen die Autorin eine künstlerische Vision des Einbruchs unterschiedlicher Naturdesaster entwickelt, die der Menschheit als Folge des Klimawandels drohen. Kassandra befürchtet das Eintreten von gewaltsamen Wettererscheinungen und leidet unter den imaginierten Bildern der Anzeichen der „Apokalypse von innen“, im Gegensatz zu Beyers Protagonisten, der - als Schriftsteller und Augenzeuge des Elbhochwassers - ein einzelnes, authentisches Naturdesaster beschreibt. Beide Autoren verbindet jedoch die Kritik an der Einwirkung medialer Bilder von Katastrophen auf die menschliche Vorstellungskraft.

Röggla stellt die Medienberichte über den Klimawandel in den Zerrspiegel der Satire und wandelt sie künstlerisch ab, um ihre Kritik an der Konsumgesellschaft zu üben: „Mich interessiert der gesellschaftliche Umgang mit Naturkatastrophen und die Erzählform darüber.“ (Warenski 2006, 16) Mit ironischen Fragmenten von Originalwetterberichten steigert die österreichische Autorin die Aussagekraft ihres Werks. Durch den Gebrauch des Fachwortschatzes aus den Wetterberichten zeigt die Autorin auf, dass die Medien die besonders sensiblen Zuschauer in eine ständige „Alarmbereitschaft“ versetzen, die jedoch angesichts der sich vertiefenden ökologischen Katastrophe angebracht zu sein scheint. Die Schriftstellerin betont zudem durch die Originalsprache des Wetterberichts, dass der Klimawandel Realität ist, auch wenn die Risikogesellschaft seine wahrnehmbaren Anzeichen ignoriert.

Bereits im Erzählband Irres Wetter, der 2000 erschien, benutzt Röggla ironisch Entlehnungen aus der meteorologischen Fachsprache. In einem literarischen Bild eines Spaziergangs zweier Protagonisten durch die deutsche Hauptstadt verwendet sie z. B. die Bezeichnung „niederschlag“. (Röggla 2000, 81) Der Erzählerin, die durch das nächtliche Berlin streift, erscheint der sternenklare Himmel als Zeichen einer nur scheinbaren Ruhe: „und in der oranienstraße schneit es auch heute nicht, es verglühen keine kometen, pardon, meteoriten über uns, kein niederschlag weit und breit, nicht einmal der lindenblütenregen, den man ansonsten in der stadt antrifft, mangels der bäume.“ (Röggla 2000, 81) Die junge Frau fühlt in der dicht bebauten Großstadt intuitiv eine unbestimmte „Bedrohung von innen“. (Nitzke 2012, 176) Der nur imaginierte Komet gilt hier als Omen für eine Katastrophe, eine „Apokalypse von innen“, die bereits im Gange ist. $^{17}$ (Vgl. Nitzke 2012, 176)

17 Marcel Beyers Text „Andere Echos“ erwähnt ebenfalls einen Meteoriten. Das Bild des leuchtenden Himmelskörpers wird hier jedoch entmythisiert. Der Erzähler bemüht sich, mysteriöse Phänomene in der Natur rational zu erklären: „[I]ch habe bloß einen verirrten Leuchtkörper 
Erst im Band die alarmbereiten wird jedoch die Umweltkatastrophe als eine der wichtigsten Ursachen des nahenden, von Menschen selbstverschuldeten Untergangs dargestellt. (Vgl. Röggla 2010a) In dieser Prosasammlung umgeht die Autorin eine naturalistische Wirklichkeitsdarstellung. Neben poetischen Bildern imaginierter Katastrophen verwendet sie einzelne fachspezifische Ausdrücke aus dem Bereich der Umweltforschung. Röggla vermeidet längere Zitate aus medialen Berichten und dokumentarisches Festhalten von Anzeichen des Klimawandels. Ihr Ziel ist es, die Sinnlosigkeit oft unverständlicher medialer Diskurse über den Klimawandel vor Augen zu führen: Die sogenannten Durchschnittsbürger äußern Zweifel, ob dieser sich tatsächlich ereignet, und stellen populäre „klimawandelvorstellungen“ in Frage. (Röggla 2010a, 36)

In den zu Monologen verkümmerten Telefonaten mit ihrer Bekannten zitiert Kassandra unverständliche mediale Berichte. Sie spricht u. a. „über das wasser mit seinen wassertemperaturen, mit seinen fließgeschwindigkeiten und seinem salzgehalt“ oder „die grassierende algensorte mit ihrer grassierenden algenblüte, die das pazifische wasser beispielsweise rot färbe aufgrund ihres eisengehalts“. (Röggla 2010a, 31) Kassandras Bekanntenkreis schenkt ihren Worten jedoch keinen Glauben. Die Risikogesellschaft versucht, den Informationsfluss der Medien bezüglich der Umweltzerstörung zu bagatellisieren. (Vgl. Röggla 2010a, 36) Eine männliche Figur im Text die ansprechbare äußert, die Katastrophengefahr werde in den Medien nur hochgespielt, um von der wirtschaftlichen Krise abzulenken. (Vgl. Röggla 2010a, 34) Die Gesellschaft streitet zudem die Tatsache ab, dass der globale Klimawandel von Menschen verschuldet ist:

das sei doch ganz normal dort, das sei immer schon so gewesen, dass es extreme gegeben habe, es habe immer schon diese extremen temperaturschwankungen gegeben, immer schon diese extremen dürren, auf die mit sicherheit gleich extreme niederschläge folgten. man müsse nur lange genug warten. auch dass landschaften versteppten, komme mal vor, dass trockenheiten sich ausbreiteten und eisflächen abnähmen, ebenfalls. das sei nur unsere naturhysterie, unsere völlig verdrehte einstellung zur landschaft, die uns aus dem konzept bringe. (Röggla 2010a, 34)

Hier nimmt Röggla kritisch Bezug auf den aktuellen Klimadiskurs und die Verschwörungstheorien bezüglich eines von manchen Politikern nur vorgetäuschten Treibhauseffekts.

In deutschlandfunk, einem weiteren Text im Erzählband die alarmbereiten, geht es um die riesigen Überschwemmungen in Mitteleuropa, die auf schwere

gesehen, ziellose Leuchtspurmunition am winterhellen Himmel.“ (Beyer 2003, 147) Kein Komet wird herbeizitiert. Der Erzähler bleibt Beobachter der ihn umgebenden Naturwelt, die er auch realitätsnah schildert. 
Gewitter folgten. (Vgl. Röggla 2010a, 182) Dieser Text hat in thematischer Hinsicht viel gemein mit Beyers „Wasserstandsbericht“, der auch eine Überschwemmung als Folge des Klimawandels zum Gegenstand hat. Skeptisch sehen beide Autoren insbesondere die Klimadebatte in den öffentlichen Medien. In überspitzter Form kritisiert Röggla durch den ironischen Gebrauch der Fachsprache sowohl den sogenannten Klima-Skeptizismus ${ }^{18}$ als auch den „klimaoptimusmus“. (Röggla 2010a, 37) Die Autorin charakterisiert zudem die „gegenmaßnahmen“, die zur Senkung der negativen Effekte des Klimawandels ergriffen werden, z. B. zur Vorbeugung von Überschwemmungen in den Niederlanden und zur Senkung der Luftverschmutzung. (Röggla 2010a, 37) Es geht für sie um Ersatzhandlungen, die die brennenden Probleme nicht lösen könnten. (Vgl. Röggla 2010a, 37) Kritisch beurteilt werden auch wissenschaftliche Konzepte, die der Erzählerin medial vermittelt wurden, z. B. das Projekt des Astronomen Roger Angel, einen künstlichen „Sonnenschirm“ zu bauen und 16.000 Milliarden durchsichtige Scheiben zwischen Sonne und Erde im Weltraum zu installieren. (Vgl. Knauer 2017)

Trotz sorgfältiger Recherchearbeit und der Verwendung von Originalzitaten, die die Sinnlosigkeit medialer Diskurse über den Klimawandel vor Augen führen, entwickelt Röggla ein zwiespältiges Verhältnis zum New Journalism. Zum Gebrauch des Dokumentarischen in der Literatur äußert sie sich häufig in ihren Essays und Interviews. Im Text Verwörterung der Welt unterstreicht die Autorin, „dass sich Autobiografismus und Dokumentarismus nicht ausschließen müssen“, und spricht sich gegen rein dokumentarische Methoden aus, denn nicht die Faktizität, sondern die Sprache der Originalberichte und das Spiel mit dem Wortschatz interessieren sie. (Röggla 2005, 83) Diese Feststellung lässt sich auch in Bezug auf den Gebrauch der meteorologischen Fachsprache in Rögglas Werk anwenden. In ihren zahlreichen Essays, Reden und Poetikvorlesungen, u.a. in Saarbrücken (2014), Essen (2014) und Paderborn (2011) (vgl. Wojno-Owczarska 2016; Canaris 2017), spricht sich die Autorin für die sinnvolle Verbindung des authentischen dokumentarischen Materials mit der kritischen Reflexion über die Wirklichkeit aus, jedoch stets bereichert durch innovative, den Leser herausfordernde Stilmittel. Ihr großes Interesse gilt besonders auch dem Zusammenspiel von Gesellschaft und Sprache; sie zeigt, wie z. B. die Idiomatik der Sprachen in Beruf und Medien unsere Alltagssprache durchdringt und unterwandert. Die Ergebnisse ihrer intensiven Recherchearbeit und die Verwendung der meteorologischen Fachsprache

18 Als ein Manifest des sogenannten Klima-Skeptizismus gilt Michael Crichtons Roman State of Fear (2004). Im Verlauf der Handlung decken die Protagonisten auf, dass die Angst vor den negativen Folgen des Klimawandels das Ergebnis einer globalen Verschwörung ist, ausgelöst durch Gewinnsucht und Machtgier. Laut Horn kritisiert der Autor die „Prognosen einer menschengemachten Erwärmung des Weltklimas als wissenschaftlich nicht belegbar“. (Horn 2014, 170) 
dienen dazu, ihre Texte als „Verbindung von Gesellschafts- und Sprachanalyse“ noch wirkungsvoller $\mathrm{zu}$ formen und $\mathrm{u}$. a. auch auf die aktuellen Klimadiskurse Bezug zu nehmen. (Allkemper 2012, 422)

In der Literaturforschung ist insbesondere nach dem Erscheinen von really ground zero (2001) die Diskussion um Rögglas Realismusbegriff entbrannt. In diesem Kontext sollte man vor allem auf die Beiträge hinweisen, die den Roman als Beispiel des semidokumentarischen Verfahrens interpretieren. (Vgl. u. a. Kaempf 2011, Wojno-Owczarska 2012 und Porombka 2015) Rögglas Realismusbegriff wurde $\mathrm{u}$. a. während der Tagungen in Berlin (Literarische Katastrophendiskurse im 20. und 21. Jahrhundert, HU Berlin, 2015, und Internationale Tagung zum Begriff der „Arbeit“, Literaturforum im Brecht-Haus, 2016), Essen (Realitätsf(r)iktionen. Kathrin Rögglas Poetik eines neuen Realismus, Universität Duisburg-Essen, 2016), Bamberg (Literatur im Ausnahmezustand, 2017), Warschau (Topographien der Globalisierung/Topographies of Globalization, 2018) und Salzburg (Kathrin Röggla und die Recherchegespenster. Realitäten in Prosa und Theater der Gegenwart, 2019) diskutiert. Nusser betont, dass Rögglas Werke „,von Realitätskonstrukten handeln, die nicht von den Texten produziert werden, sondern bei Prozessen der Kommunikation als ihre Realität außerhalb der Texte“. (Nusser 2016, 219) Gegen diese Feststellung polemisiert Canaris: „Die Diagnose ist zutreffend, jedoch wage ich zu bezweifeln, dass die von Röggla produzierten Texte außerhalb dieser Realität stehen, sie sind immer Teil des Diskurses“. (Canaris 2017, 235)

Die österreichische Autorin nimmt Bezug sowohl auf die Realität, in die sie die Umweltzerstörung einbezieht, als auch auf die aktuelle Klimadebatte. Die „Apokalypse von innen“ sieht sie nicht nur in den Folgen der Umweltzerstörung, sondern auch darin, dass sich die Klimadiskurse zwar immer weiter entfalteten, es jedoch zu keiner Lösung der verhandelten Probleme komme, begünstigt durch die Gleichgültigkeit der Gesellschaft gegenüber dem Umweltschutz. In ihrem Erzählband die alarmbereiten benutzt die Schriftstellerin Fragmente authentischer Berichte über die Folgen des Klimawandels - und die für sie eigene Sprache - in verzerrter, oft zugespitzter Form, da sie in der heutigen Welt, in der durch mediale Berichte beherrschten Wirklichkeit, eine „Umkehrung der Realitätsverhältnisse“ beobachtet, weshalb man, ihrer Meinung nach, den Realismusbegriff neu definieren müsse:

Ich habe darüber viel in ,besser wäre: keine‘, vor allem in ,disaster awareness fair` und in ,Rückkehr der Körperfresser II‘ geschrieben - die Bestellung von Drehbuchautoren ins Weiße Haus, die Vorlage des Genres für tatsächliche Terroranschläge, die Verwendung von Computerspielen für Soldatentrainings, die Mediennarrationen der Finanzkrise und die ganze übliche Katastrophengrammatik, die uns tagtäglich aus den Medien entgegentritt. (Röggla 2015, 34) 
Röggla fordert daher in ihren Saarbrücker Poetikvorlesungen die Schriftsteller zu kritischer Auseinandersetzung mit der sie umgebenen Realität auf. (Vgl. Röggla 2015) Als Sprachrohr einer Gruppe von wenigen kritischen Medienrezipienten in einer Gesellschaft des Spektakels verarbeitet sie Originalberichte, die sie in leicht abgewandelter Form in ihre Werke aufnimmt. Dieses Verfahren nennt Canaris, in Anlehnung an Röggla, das Prinzip der Schraube, „die sich immer weiter und tiefer dreht und dabei aufnimmt, was ihr auf dem Weg begegnet“. (Canaris 2017, 235) Daher schlägt die Literaturwissenschaftlerin in ihrem Beitrag in Bezug auf Rögglas Realismusbegriff die Bezeichnung „diskursiver Realismus“ vor. (Canaris 2017, 235)

Im Band die alarmbereiten finden sich Originalberichte aus unterschiedlichen Medien über Naturkatastrophen, jedoch bemüht sich die Autorin, das dokumentarische Material immer durch eine subjektive, künstlerische Komponente zu ergänzen. (Vgl. Röggla 2010a) In ihrem Schaffen betont Röggla dank ihres innovativen Erzählstils, der auch das Mittel des ihr eigenen Gebrauchs des Konjunktivs einschließt, dass der durch mediale Bilder desensibilisierte Mensch paradoxerweise infolge der Überfrachtung mit dokumentarischen Berichten sich von der Realität abgrenzt und seine Existenz fortführt, als gebe es keine ernsthafte „Bedrohung von innen“ der uns vertrauten Welt. (Nitzke 2012, 176) In der Paderborner Poetikvorlesung das stottern des realismus. fiktion und fingiertes, ironie und kritik spricht sich die Autorin erneut gegen einen „falsch verstandenen dokumentarismus“ aus. (Röggla 2011, 13) Die Künstlerin lehnt Romane ab, in denen der Autor seine Figur ,über reale schauplätze jagt und [...] nachrichten der wirklichen welt referieren lässt, damit man weiß, dass er heute und jetzt angesiedelt ist“. (Röggla 2011, 5) Die politisch engagierte Autorin glaubt an die Aufgabe des Künstlers als ein Gewissen der Gesellschaft.

Das Werk des deutschen Schriftstellers Marcel Beyer zielt ab nicht nur auf die Aufzeichnung der Folgen einer realen Katastrophe, als ein Bericht des Augenzeugen des Elbhochwassers von 2003, sondern auch auf die Präsentation der Einwirkung medialer Diskurse auf den Einzelnen. (Vgl. Beyer 2003) Vergleichbar mit dem in der Bibel prophezeiten Weltuntergang bricht in Beyers Text die Klimakatastrophe herein, auch wenn die ersten Anzeichen des nahenden Hochwassers ignoriert werden. Die Wiedergabe von authentischen Wetterberichten und die sachlichen Meldungen über den Wasserstand sollen die Authentizität des Berichts und die Gleichgültigkeit der Kräfte der Natur gegenüber menschlichen Schicksalen hervorheben: „,Gemessen um sechzehn Uhr‘, und: ,Tendenz steigend.' Gedehnte Zeit, geraffte Zeit. Selbst wenn die Pegelstände nur jede Stunde abgelesen werden, selbst wenn nicht länger abgelesen werden kann, weil der Pegel vom Wasser zerstört worden ist.“ (Beyer 2003, 42) Das Unwetter bricht wiederholt in den ruhigen Alltag der Elbanrainer herein, vergleichbar mit 
der alttestamentarischen Strafe Gottes für den menschlichen Frevel an der Natur: Alle Sünden gegen die natürliche Weltordnung werden geahndet. Daher scheint auch in Bezug auf das Bild des Klimawandels in Beyers „Wasserstandsbericht“ die Bezeichnung „Apokalypse von innen“ zutreffend. Die Katastrophe, deren Ursachen in der von Menschen herbeigeführten Umweltverschmutzung liegen, zersetzt die bestehenden Verhältnisse und stellt das gesellschaftliche Zusammenleben auf den Prüfstand. Die verspätete Reaktion seitens der Behörden, die für mangelnde Vorkehrungen gegen eine Überschwemmung der am Fluss gelegenen Gebiete verantwortlich sind, aber auch die Rolle der sensationslüsternen Medien in der Krisensituation werden hier kritisiert. Beyers Art der Darstellung von Fragen der fortschreitenden ökologischen Zerstörung unterscheidet sich klar von Rögglas Werk. Im Text „Wasserstandsbericht“ kontrastiert der nüchterne Erzählstil der medial vermittelten Berichte mit der unglücklichen Lage der Elbanrainer:

\footnotetext{
Weinend steht die junge Frau auf halber Höhe am Bahndamm [...], die Sportschuhe mit Schlamm beschmiert, die Füße im regennassen Gras [...], unentschieden, mit regennassem Haar und einem Autoschlüsseletui in ihrer ausgestreckten Hand [...]. Die Strümpfe der Frau sind naß, ihre Hose, der Saum ihrer Windjacke und die Ärmel, alles von einem schimmernden Film überzogen. (Beyer 2003, 11)
}

Der Autor kritisiert hier, in Anlehnung an Susan Sontag, die mediale Verbreitung der Luftaufnahmen von Dresden und von menschlichem Leid als Folge des Elbhochwassers. (Vgl. Sontag 2003a, 2003b und 2003c) Anders als Röggla stellt Beyer zudem die Umstände dar, die der Publikation von Katastrophenberichten vorangehen. Dass man von ihm als Augenzeugen des Naturdesasters verlangt, über das menschliche Leid, an dem er teilhat, nüchtern zu berichten und ein Pressefeuilleton über das Hochwasser in großer Eile auszuarbeiten, findet der Erzähler geschmacklos. (Vgl. Beyer 2003, 14)

Trotz des Gebrauchs unterschiedlicher Ausdrucksformen haben Beyers und Rögglas Werke einiges gemein. Der Text „Wasserstandsbericht“ stützt sich zwar auf Originalberichte, die Einarbeitung dieser Textform dient jedoch der Kritik am rücksichtslosen Handeln der Machthaber und Journalisten, die ihre Möglichkeiten nicht zur Verbesserung der Situation der Betroffenen einsetzen. Somit erfüllt hier der Wetterbericht als Nachweis für eine Naturkatastrophe eine Hilfsfunktion und ist nicht selbst Gegenstand der Erzählung. Beyers Recherchearbeit in Verbindung mit den eigenen Erlebnissen während des Hochwassers von 2003 führt jedoch zum literarischen Zusammenschluss von persönlicher Sicht und naturalistischer Wirklichkeitsdarstellung - ein Beispiel für den New Journalism.

In Beyers Erzählband Nonfiction wird zudem die Reflexion über den Gebrauch des Dokumentarischen in der Literatur angestoßen: „Ich beurteile Literatur weniger danach, ob sie mir etwas Außergewöhnliches, etwas Unbekanntes vor- 
führt, als danach, ob ich beim Lesen selbst Dinge, die mir bekannt sind, aufgrund der Sprache, der Darstellungsweise als etwas Unbekanntes, Neues wahrnehme.“ (Beyer 2003, 24) Die überzeugende Verbindung von authentischem, sachlichem Bericht mit der Darstellung von eigenen Erfahrungen lassen den Autor die Auswirkungen der Umweltkatastrophe mit sparsamen Mitteln nachvollziehbar darstellen, als eine „Bedrohung von innen“: „Texte mit spektakulärem Inhalt sind mir in gewisser Weise von vornherein suspekt.“ (Beyer 2003, 24; Nitzke 2012, 176)

In ihren Werken benutzen Beyer und Röggla Begriffe aus der Meteorologie jedoch auch metaphorisch. Einige Beispiele seien genannt: Eine weibliche namenlose Figur in Rögglas Theatertext Machthaber stellt fest, sie sei schuld am „dauerfrost“ und meint damit die ,versteinerten verhältnisse“ unter den Mitarbeitern einer Firma. (Röggla 2010b, 29) Ein bevorstehender finanzieller Bankrott wird als „komplette sonnenfinsternis“ bezeichnet. (Röggla 2010b, 37) Um schwer verständliche ökonomische Prozesse darzulegen, verwendet die Autorin bestimmte Ausdrücke, die man eher in Bezug auf natürliche Vorgänge erwartet. Eine weibliche Figur im Text Machthaber, die in einer Investmentfirma arbeitet, löst durch eine falsche Entscheidung eine „finanzlawine“ aus und bezeichnet sich als „tsunami-empfänger“, da sie die Folgen ihres Fehlers tragen muss: „ich sei das epizentrum, das kurzfristige, mittelfristige und langfristige tsunamis hervorrufe.“ (Röggla 2010b 29) Den Titel der tsunami-empfänger trägt auch ein Hörspiel von Röggla (BR 2010), in dem sie ökonomischen Katastrophen nachgeht. Die Autorin schöpft reichlich aus dem Fachwortschatz der Meteorologie und wandelt die Entlehnungen ab, um die Leistungsgesellschaft als gefühllos zu charakterisieren.

Der Titel des Prosabandes Irres Wetter ist auch als Metapher für die unvorhersehbaren Ereignisse im Leben der Betroffenen zu sehen. In Irres Wetter suchen die Teilnehmer der Berliner Love Parade im Regen vergeblich nach Spaß und passender Musik. Durch die ungünstige Wetterlage an einem Hochsommertag erfüllen sich die Erwartungen der österreichischen Touristen in Berlin nicht. Ihnen bieten sich nur der Tumult, Alkoholfahnen und der Gestank durchnässter Kleidung. (Vgl. Röggla 2002a, 5-12)

Die für Sprachspiele bekannte Autorin schöpft aus dem reichhaltigen Wortschatz der Klimaspezialisten und verwendet diese Sprachfertigkeit zum Teil ironisch, um Katastrophen in persönlichen Lebensbereichen treffend zu charakterisieren. Röggla spielt zudem mit Wetterbezeichnungen, um Gemütszustände von Figuren zu vermitteln. Im Text „Beinahe ein Leben auf der Autobahn verbracht“ korrespondieren die negativen Auswirkungen des Klimawandels mit der Enttäuschung der Erzählerin, der die Suche nach dem Lebensziel fehlgeschlagen ist. Die Identitätsfindung erscheint ihr unmöglich in Zeiten, ,in denen der winter immer häufiger kommt und der sommer zu einem beliebigen kurzzeitgedächtnis verkommt“. (Röggla 1996, 110) 
Auch Beyer benutzt die Wetterbeschreibungen zuweilen im metaphorischen Sinn, jedoch selten ironisch. Im Roman Menschenfleisch (1991) spielt die Szene der Reanimation der Hauptfigur vor dem Hintergrund eines Sonnenuntergangs; das Rot des abendlichen Himmels wird mit der Farbe des Blutes verglichen und die Gefahr des Todes mit dem Beginn der Nacht: „[J]etzt scheint die Sonne, jetzt geht das Licht aus“. (Beyer 1991, 24) Auch die Beschreibung der meteorologischen Phänomene in Beyers Nonfiction dient als Hintergrund für die Darstellung von Emotionen, z. B. in „Jenseits der alten Fotoalben“: „Es schneit. Ein trüber Tag Ende Januar 2001. Trübe genug, um die Erinnerung an trübe Zeiten wachzurufen.“ (Beyer 2003, 49) Der Gebrauch der meteorologischen Fachsprache im übertragenen Sinn ist in seiner Prosasammlung Nonfiction viel seltener als in Rögglas Erzählband die alarmbereiten. Beyers „Wasserstandsbericht“ bleibt einer der wenigen Texte in diesem Sammelband, denen das Dokumentarische, darunter der originale meteorologische Wortschatz, und auch die ökokritische Einstellung zur umgebenden Realität zugrunde liegt.

Beide Künstler reflektieren auch die Möglichkeiten, mittels modernster Technik Wetterlagen zu kontrollieren. In Beyers Gedichtband Graphit wird künstlicher Schnee in einer Skihalle dargestellt. Die Technik habe es ermöglicht, dem Winter Dauer zu verleihen. (Vgl. Beyer 2014, 9) Im Roman Kaltenburg (2008) reflektiert der Erzähler, wie man mit technischen Mitteln das Klima beeinflussen könnte, vergleichbar z. B. mit dem Einsatz von Klimaanlagen in Wohn- oder Arbeitsbereichen. (Vgl. Beyer 2008) Der Hauptprotagonist, ein Vogelforscher, erinnert zudem an den klimabedingten Substanzverlust von Bauwerken in Posen: „Welch einen Gegensatz bildet dazu die Welt der Einkaufspassagen, wie anders nimmt sie mich auf, mit ihrer sommers wie winters angenehmen Temperatur, dem Licht, den Stimmen, wo ich nicht verlorengehen kann. Ein Glasdach überspannt den Raum, oben auf den Eisenträgern sitzen Tauben.“ (Beyer 2008, 77) Die zitierte Textpassage erinnert u.a. an das Bild von klimatisierten Büroräumen in Ernst-Wilhelm Händlers Wenn wir sterben. (Händler 2011 [2002], 19) Die sterilen Räume ermöglichen dem modernen Menschen die Ausgrenzung der Realität und auch der Folgen des Klimawandels, der bereits Realität geworden ist.

Auch Röggla greift in ihren Texten die Frage auf, ob der moderne Mensch imstande ist, Wetterlagen zu beherrschen, und nimmt in diesem Zusammenhang Bezug auf die aktuelle öffentliche Debatte: „man baue nicht nur deiche in holland und dänemark, man plane auch die konstruktion von kunststoffschutzschilden, um sie in die stratosphäre zu bringen. von schwefelteilchen, die das sonnenlicht reflektieren, höre man, die sie den flugzeugabgasen beimengen wollten. von $\mathrm{Co}_{2}{ }^{-}$ endlagern unter der erde, zu denen man pipelines quer durch die bundesrepublik legen könne.“ (Röggla 2010a, 37) Röggla kritisiert hier die Einwirkung medialer 
Berichte auf die Vorstellungskraft der Fernsehzuschauer. In ihrem Werk verweist die Autorin darauf, dass in unserer „publizistischen Gesellschaft“ (Humborg und Nguyen 2018) die Grenzen zwischen Fakt und Fiktion, Realität und Inszenierung längst nicht mehr trennscharf zu ziehen sind.

\section{Fakt und Fiktion: Zur Darstellung ausgewählter Wetterphänomene in Marcel Beyers Texten}

Während für Beyers „Wasserstandsbericht“ - als Beispiel für den New Journalism ${ }^{19}$ - eine realitätsnahe Darstellung der Folgen des Unwetters charakteristisch ist, findet man in seinem Schaffen Beispiele dafür, dass der Autor meteorologischen Phänomenen eine symbolische Bedeutung verleiht, z. B. fungieren sie als Hintergrund für menschliche Gemütszustände oder als Sinnbilder der Vergänglichkeit. Wie in Rögglas Erzählband die alarmbereiten nehmen die Bürger in Beyers Erzählung die Hochwassergefahr durch die Folgen des Treibhauseffekts nicht ernst, bis es zur Katastrophe kommt, die ihren Glauben an das Bestehen der technisierten Zivilisation in Frage stellt. Der Einbruch der enormen Kräfte des Wassers reißt die Menschen aus ihrem Alltag.

Marcel Beyer stellt lebensnah die zu Realität gewordene „Apokalypse von innen“ dar. (Vgl. Nitzke 2012, 176) Die auf die Regenfälle und die erste Flutwelle folgenden Wettervorhersagen werden mit großer Anspannung erwartet. Die befürchtete Hiobsbotschaft kann für die Betroffenen eine dramatische Verschlechterung ihrer Lebensumstände bedeuten. Niemand, weder Machthaber noch die Vorsehung, könne die Durchschnittsbürger vor wütenden Wassermassen retten. Der Autor betont die Unberechenbarkeit der natürlichen Phänomene. Ausgerechnet an einem sonnigen „Hochsommertag“ bald nach der Überschwemmung zerstört der Wetterbericht die Hoffnung auf die Rückkehr zur Normalität: Eine neue Flutwelle wird angekündigt. (Vgl. Beyer 2003, 18)

Die Darstellung der Folgen des Naturdesasters, erzählt aus der Perspektive eines Elbanrainers, trägt naturalistische Züge und wirkt dank der Fragmente des Wetterberichts authentisch. Die männliche Hauptfigur wartet auf eine Erklärung

19 Einige Merkmale des New Journalism sind in Beyers Erzählung jedoch nicht berücksichtigt. So ist laut Pörksen z. B. der häufige Perspektivenwechsel für den New Journalism charakteristisch, was auf Beyers Erzählung nicht zutrifft. In Raymonds Queneaus Roman Stilübungen erscheint dagegen derselbe Inhalt in 99 verschiedenen Versionen, ,in einem jeweils anderen Berichterstattungsmuster“. (Pörksen 2004, 16) 
der Gründe für die Katastrophe durch die Medien. ${ }^{20}$ Beyer schildert hier nicht nur die Naturkatastrophe an sich, sondern reflektiert auch, ähnlich wie Röggla, die Rolle der Medienberichte über die Folgen des Klimawandels. Wie die Augenzeugen der Attentate auf das World Trade Center in Rögglas really ground zero (Röggla 2001), verspürt auch der Erzähler in „Wasserstandsbericht“ den inneren Drang, die Katastrophe zu dokumentieren. (Vgl. Beyer 2003, 13) Bei der literarischen Aufarbeitung der Elbfluten schildert Beyer jedoch eine ganz andere Situation als die der medialen Ausbeutung der Tragödie in Rögglas Roman, wo die Erzählerin von Anfang an die Übertragung der sogenannten breaking news live thematisiert. (Vgl. Röggla 2001)

Der deutsche Autor greift die Frage auf, welchen Wert heutzutage Aussagen von Augenzeugen und mediale Berichte überhaupt haben. Nur mit „Gummistiefel[n], Arbeitshandschuhe[n] und eine[r] Wasserflasche“ ausgestattet, erkundet der Erzähler in Beyers Text die überfluteten Landstriche. Ungeachtet der sachlichen Wetterberichte und der kompetent vermittelten Angaben zum Pegelstand solle man, seiner Meinung nach, als Augenzeuge vornehmlich die menschlichen Tragödien ins Blickfeld nehmen: „Ich werde Augenzeuge, ich kann nach dem Hochwasser wochenlang von nichts anderem als von dieser Erfahrung sprechen, erstatte gern ausführlich Bericht. Ich warte nur darauf, daß jemand sich nach den Hochwassertagen erkundigt.“ (Beyer 2003, 23) Der Erzähler, zunächst ohne Zugang zu den Medien, interessiert sich später für unterschiedliche Formen der Berichterstattung: „Wie hatten Berichte ausgesehen, die für die Außenwelt bestimmt waren, welche Möglichkeiten gibt es, einen Eindruck in einem festgelegten Format zu vermitteln, welchen Beschränkungen, einfach eigenen Gesetzen unterliegt die Berichterstattung im Fernsehen, in der Zeitung, im Radio?“ (Beyer 2003, 14) So sind auch in „Wasserstandsbericht“ Klimadiskurse Gegenstand der Reflexion eines Protagonisten. ${ }^{21}$

Wie Kathrin Röggla betont auch Marcel Beyer in seinem Text die Diskrepanz zwischen der Realität und deren medialem Bild: ,Jemand vom Fernsehen ruft in Dresden an, während im Fernsehen das Hochwasser in Dresden gezeigt wird [...]. Offenbar existieren zwei Welten nebeneinander, eine Bildschirmwelt und eine Welt jenseits des Bildschirmgeschehens.“ (Beyer 2003, 20) Nicht ohne Grund

20 Auch in Rögglas Theatertext fake reports verlangen die Radiohörer die mediale Interpretation der nüchternen Katastrophenberichte: ,aber sie wisse ja auch nicht, ob sie wirklich angst gehabt habe, bzw. sie habe eben nicht gewußt, ob sie angst hätte haben sollen oder nicht.“ (Röggla 2002b, 6)

21 Der Diskursanalyse Foucaults zufolge wird Wirklichkeit erst durch Diskurse erzeugt. (Vgl. u. a. Foucault 1974, 76, und Foucault 2003, 231; Burtscher-Bechter 2004) 
trägt der Erzählband den Titel Nonfiction: Trotz der Verdrängung der realen ökologischen Situation und der Folgen des Klimawandels wird der Mensch im Angesicht der Überschwemmung zur Konfrontation mit der Realität gezwungen. Nur ein abnormer Wandel der Wetterlage kann die Menschheit aus dem Zustand der inanen Gedankenlosigkeit reißen. Flutopfer sind zunächst sprachlos gegenüber den Dimensionen der Katastrophe. Das Fernsehen zeigt, „wie sie stammeln und weinen. Wie sie einen Satz beginnen, sich verhaspeln, abbrechen und hilflos mit den Schultern zucken.“ (Beyer 2003, 33) Ein Reporter formuliert einige Worte der Betroffenheit, die das Ausmaß des menschlichen Leidens nicht wiedergeben können. Die existenziellen Fragen angesichts der Gefährdung der Elbanrainer nimmt der Autor zum Anlass, sich mit der Bedrohung durch den Klimawandel auseinanderzusetzen und auf den aktuellen Realitätsdiskurs einzugehen. ${ }^{22}$ In Beyers Text versetzt der Ausnahmezustand während des Hochwassers den „Körper in Alarmbereitschaft“, vergleichbar mit dem Stress der jungen Frau im Erzählband die alarmbereiten und den Ängsten der New Yorker nach den Attentaten in really ground zero. ${ }^{23}$ (Vgl. Beyer 2003, 16; Röggla 2001 und 2010a)

22 Auch im Titel really ground zero ist der Bezug zum Realitätsdiskurs prominent. Die Augenzeugen der Katastrophe verharren in beiden Texten fassungslos. Assoziationen mit Susan Sontags Essay „Das Leiden anderer betrachten“ (2003) stellen sich hier ein. (Vgl. Sontag 2003a und 2003b) Die angespannte Situation in Dresden nach der ersten Flutwelle in Beyers „Wasserstandsbericht“ erinnert zudem an den von Röggla beschriebenen Ausnahmezustand in New York. Die Katastrophe reißt den Menschen aus seinem gewohnten Alltag: Es kreisen Hubschrauber in der Luft, der Straßen- und Zugverkehr ist eingestellt, man benutzt Notstromaggregate. (Vgl. Beyer 2003, 12) Die Hauptfiguren in beiden Texten sind Schriftsteller; doch selbst die sprachlich versierten Künstler fühlen sich ihrer Ausdruckskraft beraubt im Angesicht des Ausmaßes der realen, medial vermittelten Tragödie. Die Passagen, die das Wetter beschreiben, haben jedoch eine ganz andere Funktion im Vergleich zu Beyers Ausführungen: Während in seinem „Wasserstandsbericht“ Wetterextreme vorherrschen, ist die Schilderung der harmonischen Wetterlage in Rögglas Roman ein Zeichen der ersehnten Normalität, wenn auch beide Autoren die Gleichgültigkeit der Natur gegenüber menschlichem Schicksal betonen.

23 Niels Werber zufolge habe die Entstehung des Romans im 18. Jh. die Zielsetzungen der Literatur verändert. (Vgl. Werber 2004, 167) Das Schreibziel, Wirklichkeit zu vermitteln, habe an Bedeutung gewonnen. Seit dem 18. Jh. gelte auch der Roman als Erzählform für „die Vermittlung komplexer Weltentwürfe“ und bleibe ,an den Wunsch der Autoren nach komplexer Weltvermittlung geknüpft“, ein Ansatz, der sich laut Dieter Wellersdorf als „Utopie der Schriftsteller“ erweise. (Vgl. Bleicher 2004, 30; Wellersdorf 1988, 15) Bleicher stellt fest: „Bereits die von Heinrich Heine und Ludwig Börne geprägte Literatur des Vormärz versuchte bisherige Grenzen zwischen Kunst und Politik, Fiktion und aktueller Wirklichkeit zu durchbrechen. Dieser Veränderung ging eine Kritik an der fiktiven Welt der Poesie mit ihrer rein ästhetischen Orientierung und ihrem Mangel an aktuellem Wirklichkeitsbezug voraus. Eine vergleichbare Situation ist in der deutschsprachigen Literatur der 1980er und 1990er Jahre erkennbar.“ (Bleicher 2004, 32) Laut Canaris sei 
Auch in seinen Gedichten, z. B. im Band Graphit (2014), reflektiert Beyer zuweilen die aktuellen Klimadiskurse und betont den Unterschied zwischen der (realen) Naturwelt und deren medialen Bildern. In einem dieser Texte verweist der Dichter auf die Darstellung eines Schneesturms in Sergej Eisensteins Film Alexander Newski (1938). (Vgl. Beyer 2014, 10) Das lyrische Ich erinnert an die Schwierigkeit, den Eindruck einer winterlichen Landschaft mitten im Sommer in einer Filmproduktion wiederzugeben: Die Bühnenbilder hätten in Eisensteins Film ein ,lichtaufsaugendes Gemisch / aus Naphtalin und Kreide“ benutzt. (Beyer 2014, 9) Somit können auch Wetterphänomene für die voyeuristische Gesellschaft vorgetäuscht werden. Auch in diesem Gedicht steht das Verhältnis zwischen Fakt und Fiktion im Zentrum. Beyer führt hier unterschiedliche Zeitebenen ein und verweist darauf, dass Wetterberichte heutzutage nicht mehr notwendig seien, in einer Zeit, in der man z. B. durch Webcams informiert wird. (Vgl. Beyer 2014, 7)

In seinem Roman Spione (2000), in dem der Erzähler u.a. Geheimnisse aus der Vergangenheit seiner Vorfahren dank alter Fotos entdeckt, fungiert der Schnee, der über die Asche verbrannter Bilder fällt, als Symbol des Vergessens und der inneren Ruhe; insbesondere steht der Schneefall für die Verdrängung von tragischen Ereignissen aus der Zeit des Zweiten Weltkriegs, für die verblassenden Erinnerungen an die verstorbenen Großeltern des Erzählers, das Leugnen von Familiengeheimnissen und den Schlussstrich zwischen Vergangenheit, Gegenwart und Zukunft. (Vgl. Beyer 2010) Das Werk greift die Frage auf, ob man Bilder aus einer Welt voller Gewalt weiter aufheben und die Erinnerungen daran auf diese Weise bewahren sollte. Der Erzähler stellt fest: „Heute käme ich nie auf die Idee, etwas zu photographieren.“ (Beyer 2010, 82) Alte Fotoalben, die die Familiengeschichte des Protagonisten abbilden, aber auch andere „Erinnerungsstücke“ wie Opernprogramme, Libretti und Partituren werden in Beyers Roman vernichtet als Zeichen einer Zivilisation, deren Errungenschaften durch die tragischen Ereignisse der beiden Weltkriege fraglich geworden sind. ${ }^{24}$ (Vgl. Beyer 2010, 76, 108-111, 309 und 328) Unangenehme Wahrheiten über die Geschichte der eigenen Vorfahren werden verdrängt, was den Verzicht auf die Klärung der eigenen Identität bedeutet. (Vgl. Beyer 2010, 30) Die belastenden historischen Ereignisse sollen in Vergessenheit geraten, symbolisch überhöht im Bild des reinen Schnees, der die Asche der verbrannten alten Fotos gnädig bedeckt: „Der

der literarische Realismus ,in den letzten Jahren in der Forschung ein durchaus kontrovers und kritisch diskutierter Begriff [...], dennoch ist er etablierter [Terminus], um die problematische Beziehung zwischen Literatur und Wirklichkeit zu benennen.“ (Canaris 2017, 233; vgl. Link 2008, 6) 24 An anderer Stelle schildert der Autor u. a. auch eine „Kamera am Flugzeugrumpf“ während eines Bombenabwurfs. (Vgl. Beyer 2010, 193). 
Schneefall wird dichter, ich gehe hinaus, der Schneefall fällt weich, ich sehe die Kuppen meiner Schuhe, ich sehe, es wird Schneeränder geben. “(Beyer 2010, 110) Nicht die Wetterlage oder die alltäglichen Ereignisse werden hier thematisiert, im Zentrum steht die Frage nach der Vergänglichkeit unserer Spuren in der Welt, verdeutlicht auch im folgenden Bild:

Meine Hand, wie sie sich in den Schnee senkt, den frisch gefallenen, darunter Eis, was über Tag geschmolzen und zur Nacht wieder gefroren ist. Meine gespreizte rechte Hand, auf ihrem Rücken sind zwei dunkle Punkte schwach erkennbar, Warzen vielleicht, beim ersten Glied des Zeigefingers eine kurze Narbe, von einem Biß her, ungefähr mit elf, der breite Handrücken ist nur an der Seite behaart, und keine Adern, keine Flecken.

Ich nähere mich dem Boden langsam, meine fünf Fingernägel geben einen Abdruck im Schnee vor meinen Schuhen. (Beyer 2010, 111)

Kennzeichnend ist, dass Beyer in seiner Prosa oft winterliche Szenen schildert, was u.a. mit der Anwendung der chiaroscuro-Technik, der gezielten Gegenüberstellung von Schwarz und Weiß, verbunden ist. ${ }^{25}$ Die Wetterlagen, die in Beyers Roman Spione auftreten, korrespondieren jedoch auch mit seinem Zukunftspessimismus, insbesondere im folgenden Bild der kalten Tage in Schweden:

Über Tag habe ich immer wieder von neuem das Gefühl, es werde jeden Moment dunkel, morgens im Schneehimmel ein Sonnenschimmer, und als ich nach dem Frühstück auf die Straße trat, kam es mir so vor, als seien mehrere Stunden unbemerkt an mir vorübergezogen, nun folge schon der Sonnenuntergang [...]. Den ganzen Winter herrscht ein solcher Dämmer, es heißt, bei manchen Einheimischen führe er zu tiefer Niedergeschlagenheit. Möglich, ich konnte den Zustand nicht lange ertragen. (Beyer 2010, 273)

25 Im Reisetagebuch „Andere Echos“ zeichnet der Erzähler ein Bild der Stadt Klaipeda (Litauen) mit Kontrasten von Helligkeit und Dunkel: „[D]ie Sonne scheint, Schnee liegt [...], die Lastenkräne rostig, dunkel gegen den Hintergrund der Stadt“. (Beyer 2003, 146) Hier sind Parallelen zu finden u.a. zu Friederike Mayröckers „Reise durch die Nacht“, William Faulkners Absalom, Absalom!, Junichiro Tanizakis Die geheime Geschichte des Prinzen Musashi und Claude Simons Geschichte. (Vgl. Beyer 2003, 91 und 94) Auch im Text „Das sowjetische Apartment“ richtet sich das Hauptaugenmerk des Erzählers auf den Gegensatz zwischen Licht und Dunkelheit: „Das Licht verändert sich jeden Tag von neuem, die Sonne, halbe Sonne oder auch bloß der dunstige Schimmer im langen Winter erfaßt den Tisch, die Schränke, die dunklen Vorhänge und den Bodenbelag.“ (Beyer 2003, 183) Der Autor schildert Lichtverhältnisse in verschiedenen Jahreszeiten und unterschiedlichen Ländern: „Der sommers wie winters frühe Sonnenuntergang in Marrakesch“ und das „Winterlicht in Stockholm“. (Beyer 2003, 91) Dem Schriftsteller liegt in seiner Prosa an der Bedeutung des Lichts für die Gestaltung der Handlung und nicht an der Schilderung verschiedener Wetterlagen und Naturphänomene: „Die Lichtverhältnisse führen zu einer Szene, unbewußt, die Lichtverhältnisse lassen Szenen entstehen“. (Beyer 2003, 98) 
Der Roman Spione zeigt selten angenehme Wetterlagen. Fotos der verstorbenen Großmutter erinnern den Erzähler jedoch an schöne vergangene Tage bei mildem Frühlingswetter und christlicher Osterfreude:

Von Tag zu Tag ist es nun länger hell, es wird wärmer, bald werden die Osterferien beginnen. Langsam erholt der Boden sich vom Frost, die grauen, ausgelaugten Areale sind mit Grün durchsetzt. Das nächste Bild zeigt eine Wiese, das Gras ist hoch gewachsen, das Kleid der Frau wird von den Büschen fast vollständig verdeckt, man sieht nur ihre Schultern, ihren Kopf, den Sommerhut. (Beyer 2010, 326)

In einer poetischen Vision lässt der Erzähler das erste Treffen seiner Großeltern ablaufen. Für das Liebespaar spielen Zeit und Raum, Temperatur und Wetter keine Rolle: „Möglich, es regnet nun, ein warmer Niesel, der die beiden wer weiß wie lange schon benetzt. Möglich, es ist noch immer Hitze, die Sonne steht viel tiefer als noch eben, die Ähren werfen Schatten aufs Gesicht.“ (Beyer 2010, 63) Der Beginn des Zweiten Weltkriegs bricht in ihr ruhiges Leben ein; bald verbindet sie nur noch der „Briefverkehr“, ein unregelmäßiger Briefwechsel ohne jegliche Hinweise auf aktuelle Wetterlagen. (Beyer 2010, 181) In seinen Briefen darf der Mann, ein Offizier der Luftwaffe, keine Informationen über „die derzeitige Witterung“ vermitteln:

[Die Braut] könnte glauben, er wüßte selbst nicht so genau, wo er gerade ist, was er tut, und auf das Wetter habe er offenbar seit Tagen nicht geachtet. An keiner Stelle wechselt ihr Verlobter mittendrin das Thema, bricht er einen Gedanken unvermittelt ab, weil ihm bewußt geworden ist, er muß sich hüten, denn andernfalls wird dieser Brief seine Verlobte nie erreichen. (Beyer 2010, 181)

Der Erzähler schildert zudem als Besonderheit der Kriegszeit, dass Wetterberichte bei den betroffenen Menschen kaum Beachtung finden: Man fürchtet keine Gewitter mehr, sondern den „Schattenriß eines Flugzeugs“ am Himmel. (Beyer 2010, 193) Alles deutet darauf hin, dass die Zivilisation in eine Sackgasse geraten ist.

Der auf alten Fotos vermittelte Einklang von Mensch und Natur scheint in der Nachkriegszeit nicht mehr da zu sein. Der Zweite Weltkrieg hat das gewohnte Leben in einer Zivilisation verändert. Bei aller Erleichterung über die neu gewonnene Friedenszeit fällt dem Erzähler jedoch die zunehmende Verstädterung und Industrialisierung des Landes auf: „Wir fahren durch die Hügel und die Felder, wo sich die Landstraße schnurgerade bis zum nächsten Waldstück zieht. Wir durchqueren Ortschaften, deren Namen mir nichts sagen, kommen an zukünftigen Gewerbeparks vorbei, Asphalt und Fußwege, Bogenlampen, sonst nichts.“ (Beyer 2010, 248) Die zunehmende Gesichtslosigkeit des menschlichen Lebensraumes verändert die Lebensbedingungen der von ihm bedrohten Tierwelt: Die steigende Verschmutzung und Technisierung der Umwelt macht auch sie zu Opfern. Tradi- 
tionelle Jagd- und Fischereihandwerke gehen verloren; der moderne Bedarf an Beutetieren übersteigt bei Weitem das für Ernährungszwecke Notwendige. (Vgl. Beyer 2010, 277) Beängstigende Veränderungen betreffen auch das niedere Pflanzenreich. Als Kind hat der Erzähler interessiert Schimmelpilze beobachtet; nun warnen die Medien vor zahlreichen „Schimmelsporen“ in seinem Wohnumfeld, die „dicht wie Schneefall über dem Hügel“ schwebten. (Beyer 2010, 19 und 278) Offensichtlich ist als Folge einer Deregulierung von Ökosystemen eine neue, für den Menschen gefährliche Pflanzenart entstanden, die durchaus als bedrohliche Folge des Klimawandels gesehen werden kann. Ein unbeschwerter Spaziergang an der frischen Luft ist bei diesem Ausnahmezustand der Natur kaum mehr möglich: „,Eigentlich sollen die Anwohner nur noch mit Mundschutz vor die Tür. Alle hier haben solche Atemmasken im Haus. Aber auf die Dauer hält man sich doch nicht daran. “" (Beyer 2010, 252) Auch in diesem Werk betont der Autor die negativen Folgen der Umweltzerstörung, einer „Apokalypse von innen“, deren erste Anzeichen sich bereits bemerkbar machen und die allmählich ansteigen. (Nitzke 2012, 176)

Beyers Reflexion über den Klimawandel und kaum beherrschbare Wetterlagen vollzieht sich auf vielen Ebenen und beeinflusst die Metaphorik in seinem Schaffen. Anders als in Rögglas Werken korrespondieren in Beyers Texten stimmungsvolle Bilder der aktuellen Wetterlage mit den Gemütszuständen seiner Protagonisten. Der Autor gebraucht den meteorologischen Fachwortschatz seltener ironisch, wenn er auch oft den Bezeichnungen natürlicher Phänomene symbolische Bedeutung verleiht.

\section{Zusammenfassung}

Als Auswirkungen des Klimawandels werden in Beyers Text „Wasserstandsbericht“ (reale) heftige Regenfälle und darauf folgende Überschwemmungen gesehen; in Rögglas Erzählband die alarmbereiten nimmt Kassandra die „Bedrohung von innen“ wahr und imaginiert verschiedene Untergangsvisionen, u.a. durch eine Sintflut ausgelöste Desaster. (Nitzke 2012, 176) Beide Autoren sehen die prekäre menschliche Existenz in einer Welt der nicht mehr abwendbaren Klimakatastrophe. In ihrem Schaffen greifen Beyer und Röggla das Motiv des Klimawandels auf, einer „Apokalypse von innen“, in der sich „natürliche und zivilisatorische Ursachen nicht mehr voneinander unterscheiden lassen“. (Nitzke 2012, 176; vgl. Horn 2014, 160)

Beide Künstler beziehen zudem Stellung in der aktuellen Realismusdebatte. Fotos von der Zerstörung durch die Elbfluten und Bilder der Terrorattacken in 
New York, die in Fernsehen und Internet ausgestrahlt wurden, haben auch symbolischen Charakter. Ereignisse dieser Art sind, laut Baudrillard, Wirklichkeit und Fiktion zugleich. Der französische Soziologe und Medientheoretiker stellt in Bezug auf die Ereignisse des 11. September 2001 fest: „In einem so außergewöhnlichen Ereignis findet auch eine Wechselsteigerung von Wirklichkeit und Fiktion statt. Kein Verlust am Realen also, sondern im Gegenteil, ein Mehr am Realen, verbunden mit einem Mehr an Fiktion. Wir haben es hier mit einer total symbolischen Sache zu tun.“ (Baudrillard 2002, 69 f.) In ihrer Darstellung der furchterregenden Bilder des Leidens und der Zerstörung, die von den globalen Medien vermittelt werden, knüpfen Beyer und Röggla an Originalberichte in Fernsehen und Rundfunk an mit der nachvollziehbaren Intention, die Immanenz der Zerstörung unserer Lebenswelt vor Augen zu führen; daher nähern sich beide Künstler in ihrer Wortwahl den Stilmitteln des New Journalism. Sie nehmen Bezug auf die mediale Präsentation der realen Katastrophen und greifen die Frage auf, ob man angesichts der Entwicklung der globalen Medien den Realismusbegriff neu definieren müsse, insbesondere in einer Zeit, in der viele Szenarien der Katastrophenfilme „erfahrbar“ werden. Als Beispiele verweisen sie nicht nur auf die Berichterstattung über die Terrorattacken in breaking news live, sondern auch auf Fotos der gewaltigen Naturdesaster weltweit, der überfluteten Areale, der endlosen Wüsten und der verbrannten Baumstümpfe. Die mediale Bilderflut, die die Sensationslust der heutigen Menschen sättigen soll, dringt in den gewohnten Alltag der Medienkonsumenten ein, erregt jedoch immer seltener die Aufmerksamkeit der breiten Massen.

Beyer und Röggla betonen, dass das ständige Ausreizen von Themen im Umfeld des Klimawandels in den globalen Medien die Sensibilität der Menschen abgestumpft hat. Nicht einmal drastische Bilder von Folgen der Naturkatastrophen, die an die Visionen der biblischen Apokalypse erinnern, können als Dokumente des Schrecklichen wahrgenommen werden. In einer Welt des ,permanenten Ausnahmezustands“, in der die Entwicklung der Zivilisation als immer abwegiger erlebt wird, scheint die Vorstellung vom letzten Menschen bzw. von der selbstverschuldeten Ausrottung der Gattung Mensch immer überzeugender. Wir leben im Zeitalter des Anthropozän: Die Welt wird von den Folgen des Treibhauseffekts beherrscht, die tief in den Alltag der sogenannten Durchschnittsbürger einschneiden und das Ende des Lebens auf unserem Planeten nach sich ziehen können.

In einer schnelllebigen desensibilisierten Gesellschaft bewegen Beyer und Röggla ihre Leser zur Reflexion über die Folgen des Klimawandels und ,die selbst verschuldete Zerstörung der Natur [...], eine Bedrohung von innen“. (Nitzke 2012, 176) Zudem verweisen sie darauf, dass in Zeiten der ökonomischen Unsicherheit und mangelnder persönlicher Kontakte sich der moderne Mensch an die Vor- 
hersagen des jeweiligen Wetterberichts klammert und sie als eine Art Scheinsicherheit für die nächsten Tage betrachtet. Die Darstellung von Wetterlagen in den analysierten Texten von Beyer und Röggla scheint daher nicht nur auf die Realität der sichtbaren Folgen des Klimawandels hinzudeuten. Der Wetterbericht erweist sich oft als eine Form der Heraufbeschwörung einer Zukunft in einer Welt ohne erkennbare Merkmale realer Wirklichkeit und klar definierter Formen. So ist demnach auch der metaphorische Gebrauch von ausgewählten Fachbegriffen aus der Meteorologie in ihren Texten zu erklären, wobei man betonen sollte, dass Röggla diesen Fachwortschatz eher ironisch gebraucht, während Beyer Bezeichnungen für die Wetterphänomene in poetischen Bildern symbolisch einsetzt. Beide Autoren reflektieren auch die Möglichkeit, in Zeiten des Treibhauseffekts künstliche Klimabedingungen in abgeriegelten Gebäuden zu schaffen. Dagegen verschließt sich Rögglas Protagonistin Kassandra, völlig verängstigt, in ihrem Zuhause als die Seherin, der niemand Glauben schenkt. Die pessimistische Aussage dieser Texte setzt voraus, dass die Klima-Apokalypse, von Menschen für ihre Nachkommen - die letzten Menschen - produziert, bereits ihre ersten Opfer fordert. Der Kulturhistoriker Wolfgang Behringer betont in seiner Kulturgeschichte des Klimas: „Die Zukunft ist schwer vorhersagbar. Seriöse Wissenschaftler sollten sich hüten, in die Rolle des Nostradamus zu schlüpfen“. (Behringer 2011, 287) Der engagierten Literatur, als deren Vertreter man Beyer und Röggla betrachten kann, kommt in der Zeit der nicht bewusst wahrgenommenen „Apokalypse von innen“ als Folge der ökologischen Zerstörung die wichtige Aufgabe zu, der gedankenlosen Gesellschaft die Gefahr der Selbstvernichtung bewusst zu machen. (Nitzke 2012, 176)

\section{Literaturverzeichnis}

Allkemper, Alo. „Kathrin Röggla: ,stottern““. Poetologisch-poetische Interventionen: Gegenwartsliteratur schreiben. Hg. Alo Allkemper, Norbert Otto Eke und Hartmut Steinecke. München: Fink, 2012. 417-430.

Anders, Günther. Die Antiquiertheit des Menschen. Bd. I. München: C. H. Beck, 1956. Anders, Günther. Die Antiquiertheit des Menschen. Bd. II. München: C. H. Beck, 1980. Balint, luditha, Tanja Nusser und Rolf Parr (Hg.). Kathrin Röggla. München: text + kritik, 2017. Behringer, Wolfgang. Kulturgeschichte des Klimas: Von der Eiszeit bis zur globalen Erwärmung. München: C. H. Beck, 2011.

Beutel, Carolin. „,Eine Zeit, die Katastrophen produziert‘. Die panische Gegenwart: Kathrin Röggla sprach mit Carolin Beutel über ihr neues Buch ,die alarmbereiten', über Medienrealität, Katastrophengrammatik und einen permanenten Ausnahmezustand“. Der Standard (6447), 06.04.2010: 15.

Beyer, Marcel. Das Menschenfleisch. Frankfurt/M.: Suhrkamp, 1991. 
Beyer, Marcel. Flughunde. Frankfurt/M.: Suhrkamp, 1996 [1995].

Beyer, Marcel. Graphit. Berlin: Suhrkamp, 2014.

Beyer, Marcel. Kaltenburg. Frankfurt/M.: Suhrkamp, 2008.

Beyer, Marcel. Nonfiction. Berlin: DuMont, 2003.

Beyer, Marcel. Spione. Frankfurt/M.: Suhrkamp, 2010 [2000].

Bleicher, Joan Kristin. „Intermedialität von Journalismus und Literatur. New Journalism aus literaturwissenschaftlicher Perspektive“. Grenzgänger: Formen des New Journalism. Hg. Bernhard Pörksen und Joan Kristin Bleicher. Wiesbaden: Verlag für Sozialwissenschaften, 2004. 29-39.

Boyle, Thomas Coraghessan. A Friend of the Earth. New York: Viking Press, 2000.

Brandt, Marion und Andrzej Kątny (Hg.). Die Natur und andere literarische Orte. Festschrift für Professor Marek Jaroszewski zum 65. Geburtstag. Gdańsk: Wydawnictwo Uniwersytetu Gdańskiego, 2008.

Brandt, Renate. Porträt Marcel Beyer. Bonn: Edition Böttger, 2017.

Bühler, Benjamin und Stefan Willer (Hg.). Futurologien. Ordnungen des Zukunftswissens. Paderborn: Wilhelm Fink, 2016.

Burtscher-Bechter, Beate. „Diskursanalytisch-kontextuelle Theorien“. Einführung in die Literaturtheorie. Hg. Martin Sexl. Wien: UTB Literaturwissenschaft, 2004. 257-286.

Butler, Octavia. The Parable of the Sower. New York: Four Walls Eight Windows, 1993.

Canaris, Johanna. „,,das prinzip der schraube‘: Kathrin Rögglas diskursiver Realismus“. Gegenwartsliteratur. Ein germanistisches Jahrbuch / A German Studies Yearbook (16), 2017: 233-255.

Climate Change, Complexity, Representation. Guest ed. Hannes Bergthaller. Metaphora. Journal for Literature Theory and Media, (2) 2017.

Crutzen, Paul J. „Geology of Mankind“. Nature 415 (2002): 23.

Dombrowsky, Wolf R. und Ursula Pasero. Wissenschaft, Literatur, Katastrophe. Opladen: VS Verlag für Sozialwissenschaften, 1995.

Dürbeck, Gabriele. Stereotype Paradiese. Ozeanismus in der deutschen Südseeliteratur 1815-1914. Tübingen: Max Niemeyer, 2007.

Dürbeck, Gabriele, Bettina Gockel und Susanne B. Keller (Hg.). Wahrnehmung der Natur, Natur der Wahrnehmung. Studien zur Geschichte visueller Kultur um 1800. Dresden und Amsterdam: Verlag der Kunst, 2001.

Dürbeck, Gabriele, Christine Kanz und Ralf Zschachlitz. „Ökokritische Perspektiven und Anthropozän-Diskurs in der deutschsprachigen Literatur - eine Einleitung“. Ökologischer Wandel in der deutschsprachigen Literatur des 20. und 21. Jahrhunderts. Hg. Gabriele Dürbeck, Christine Kanz und Ralf Zschachlitz. Frankfurt/M. et al.: Peter Lang, 2018. 7-26.

Dürbeck, Gabriele und Jonas Nesselhauf. Repräsentationsweisen des Anthropozän in Literatur und Medien. Berlin: Peter Lang, 2019.

Dürbeck, Gabriele und Peter H. Feindt. „,Der Schwarm“ und das Netzwerk im multiskalaren Raum. Umweltdiskurse und Naturkonzepte in Schätzings Ökothriller“. Ökologische Transformationen und literarische Repräsentationen. Hg. Maren Ermisch, Ulrike Kruse und Urte Stobbe. Göttingen: Universitätsverlag, 2010. 213-230.

Dürbeck, Gabriele, Urte Stobbe, Hubert Zapf und Evi Zemanek (Hg.). Ecological Thought in German Literature and Culture. Lanham: Lexington Books, 2017.

Ermisch, Maren, Ulrike Kruse und Urte Stobbe (Hg.). Ökologische Transformationen und literarische Repräsentationen. Göttingen: Universitätsverlag, 2010. 
Fekadu, Sarah, Tobias Döring und Hanna Straß-Senol (Hg.). Meteorologies of Modernity: Weather and Climate Discourses in the Anthropocene. Yearbook of Research in English and American Literature (REAL) 33, 2017.

Foucault, Michel. Die Ordnung der Dinge. Frankfurt/M.: Suhrkamp, 1974.

Foucault, Michel. Schriften zur Literatur. Frankfurt/M.: Suhrkamp, 2003.

Garrard, Greg. Ecocriticism. London und New York: Routledge, 2004.

Garrard, Greg. „Ian McEwan's Next Novel and the Future of Ecocriticism“. Contemporary Literature 50.4 (2009): 695-720.

Georgopoulou, Eleni. Abwesende Anwesenheit. Erinnerung und Medialität in Marcel Beyers Romantrilogie ,Flughunde', ,Spione‘ und,Kaltenburg‘. Würzburg: Könighausen \& Neumann, 2012.

Gerste, Roland D. Wie das Wetter Geschichte macht: Katastrophen und Klimawandel von der Antike bis heute. Stuttgart: Klett-Cotta, 2015.

Glotfelty, Cheryl. „Introduction“. The Ecocriticism Reader: Landmarks in Literary Ecology. Hg. Cheryl Glotfelty und Harold Fromm. Georgia und Athens: The University of Georgia Press, 1996. XXV-XXXVII.

Goodbody, Axel. Literatur und Ökologie. Amsterdam und Atlanta: Rodopi, 1998.

Grewe-Vollp, Christa. ,Natural spaces mapped by human minds': Ökokritische und ökofeministische Analysen zeitgenössischer amerikanischer Romane. Tübingen: Narr, 2004.

Grucza, Franciszek, Willi Bolle, Gabriele Dürbeck et al. (Hg.). Akten des XII. Internationalen Germanistenkongresses Warschau 2010 - Vielfalt und Einheit der Germanistik weltweit. Bd. XIV. Frankfurt/M.: Peter Lang, 2012.

Haman, Christof (Hg.). Marcel Beyer. München: text + kritik, 2018.

Händler, Ernst-Wilhelm. Wenn wir sterben. Frankfurt/M.: Fischer, 2011 [2002].

Herring, Horace und Steve Sorrell (Hg.). Energy Efficiency and Sustainable Consumption. The Rebound Effect. Basingstoke: Palgrave Macmillan, 2009.

Hille, Almut (Hg.). Globalisierung, Natur, Zukunft erzählen. Aktuelle deutschsprachige Literatur für die Internationale Germanistik und das Fach Deutsch als Fremdsprache. München: ludicium, 2015.

Hofer, Stefan. Die Ökologie der Literatur. Eine systemtheoretische Annäherung mit einer Studie zu Werken Peter Handkes. Bielefeld: transcript, 2007.

Hoffman, Susanna M. und Anthony Oliver-Smith (Hg.). Catastrophe \& Culture. The Anthropology of Disaster. Santa Fe und Oxford: School of American Research Press, 2002.

Hoffstadt, Christian. „Über die Aktualität des Weltuntergangs“. Aus Politik und Zeitgeschichte. 62.51-52 (2012): 26-31.

Horn, Eva und Peter Schnyder (Hg.). Romantische Klimatologie. Bielefeld: transcript, 2016.

Horn, Eva. „Die Zeit des Klimas. Zur Verzeitlichung der Natur in der literarischen Moderne“. http://www.aesthetische-eigenzeiten.de/projekt/klima/beschreibung/ (30. November 2017).

Horn, Eva. Zukunft als Katastrophe. Frankfurt/M.: Fischer, 2014.

Horn, Eva. „Die Zukunft der Dinge“. Behemoth. A Journal on Civilisation 4/2, 2011: 26-57.

Humborg, Christian und Thuy Anh Nguyen (Hg.). Die publizistische Gesellschaft. Journalismus und Medien im Zeitalter des Plattformkapitalismus. Wiesbaden: Springer Fachmedien, 2018.

Hummitzsch, Thomas. „Klimawandel und Migration“. http://www.bpb.de/gesellschaft/ migration/kurzdossiers/57257/klimawandel-und-migration (28. September 2017). 
Ivanovič, Christine. „Bewegliche Katastrophen, stagnierende Bilder. Mediale Verschiebungen in Kathrin Rögglas Roman ,really ground zero““. Kultur \& Gespenster 2 (2006): 108-117.

Jabłkowska, Joanna. „Weltkatastrophen und Globalisierung“. Literatur und Literaturwissenschaft im Zeichen der Globalisierung. Hg. Anna Kochanowska-Nieborak und Ewa Ptomińska-Krawiec. Frankfurt/M. et al: Peter Lang, 2012. 29-41.

Jachimowicz, Aneta, Alina Kuzborska und Dirk H. Steinhoff (Hg.). Imaginationen des Endes. Frankfurt/M. et al: Peter Lang, 2015.

Kaempf, Simone. „Realfiktionen. Kathrin Röggla.“ Fictions/Realities. New Forms and Interactions. Hg. Jörg von Brincken, Ute Gröbel und Irene Schulzki. München: Meidenbauer, 2011. 117-120.

Kelley, Colin P. et al. Climate change in the Fertile Crescent and implications of the recent Syrian drought. Washington: University of California und Columbia University, 2015, http://www. pnas.org/content/112/11/3241.full (11. Dezember 2015).

Kersten, Jens. Das Anthropozän-Konzept. Baden-Baden: Nomos, 2014.

Klein, Christian (Hg.). Marcel Beyer: Perspektiven auf Autor und Werk. Stuttgart: J.B. Metzler Verlag, 2019.

Klein, Naomi. Die Schock-Strategie: Der Aufstieg des Katastrophen-Kapitalismus. Frankfurt/M.: Fischer, 2007a.

Klein, Naomi. The Shock Doctrine: The Rise of Disaster Capitalism. New York: Metropolitan Books/Henry Holt, 2007b.

Knauer, Roland. „All die spacigen Ideen zur Rettung der Erde“. https://www.welt.de/ sonderthemen/energiewende/article165955093/All-die-spacigen-Ideen-zur-Rettung-derErde.html (26. Juni 2017).

Krüger, Jonas Torsten. „unter sterbenden bäumen“. Grüne Literaturgeschichte. Marburg: Tectum, 2001.

Kupczyńska, Kalina. „Hinhören, weghören, aufhören - mediale und diskursive Bewegungen in ,Die Alarmbereiten“ von Kathrin Röggla. "Reise und Raum. Ortsbestimmungen der österreichischen Literatur. Hg. Arnulf Knafl. Wien: Praesens, 2014. 160-172.

Leggewie, Claus und Harald Welzer. Das Ende der Welt, wie wir sie kannten. Klima, Zukunft und die Chancen der Demokratie. Frankfurt/M.: Fischer, 2011.

Lessing, Doris. Mara and Dann: An Adventure. London: Flamingo, 1999.

Link, Jürgen. „,Wiederkehr des Realismus“ - aber welches? Mit besonderem Bezug auf Jonathan Littel.“ kultuRRevolution. zeitschrift für angewandte diskurstheorie 54 (2008): 6-21.

Link, Jürgen und Rolf Parr. „Semiotik und Interdiskursanalyse“. Neue Literaturtheorien. Eine Einführung. Göttingen: Vandenhoeck \& Ruprecht, 3. Auflage 2005 (1990). 108-133.

Link, Jürgen und Ursula Link-Heer. „Diskurs, Interdiskurs und Literaturanalyse“. Zeitschrift für Literaturwissenschaft und Linguistik 20.77 (1990): 88-99.

Löffler, Sigrid. „Melancholie ist eine Form des Widerstands“. Text \& Kritik 158 (2003): 103-111. Matthes, Frauke. „Ethical Encounters with Nature: Ilija Trojanow’s ,EisTau““. Gegenwarts-

literatur. Ein germanistisches Jahrbuch. A German Studies Yearbook 15 (2016): 311-336.

Marx, Friedhelm und Julia Schöll. Literatur im Ausnahmezustand. Beiträge zum Werk Kathrin Rögglas. Würzburg: Königshausen \& Neumann, 2019.

Mayer, Silvia. „Klimawandelroman“. Ecocriticism. Hg. Gabriele Dürbeck und Urte Stobbe. Köln, Weimar, Wien: Böhlau, 2015. 233-244.

McCarthy, Cormac. The Road. New York: Alfred A. Knopf, 2006.

McEwan, Ian. Solar. New York: Anchor, 2010. 
Murphy, Patrick D. Farther Afield in the Study of Nature-Oriented Literature. Charlottesville: University of Virginia Press, 2000.

Nitzke, Solvejg. „Animal Spirits unleashed. Kapitalistische Monster und die Zukunft ohne Markt in Dietmar Daths ,Die Abschaffung der Arten““. Monster \& Kapitalismus. Zeitschrift für Kulturwissenschaft. Hg. Till Breyer, Rasmus Overthun, Philippe Roepstorff-Robiano und Alexandra Vasa. Bielefeld: transcript, 2017. 71-84.

Nitzke, Solvejg. „Apokalypse von innen. Die andere Natur-Katastrophe in Frank Schätzings ,Der Schwarm“ und Dietmar Daths ,Die Abschaffung der Arten““. Katastrophen. Konfrontationen mit dem Realen. Hg. Solvejg Nitzke und Mark Schmitt. Essen: Christian A. Bachmann, 2012.167-188.

Nitzke, Solvejg. „Das große Unsichtbare. Die Modellierung von Klima zwischen Wissenschaft und Literatur“. Forum Interdisziplinäre Begriffsgeschichte 5.1 (2016): 90-101.

Nitzke, Solvejg und Nicolas Pethes (Hg.). Imagining Earth. Concepts of Wholeness in Cultural Constructions of Our Home Planet. Bielefeld: transcript, 2017.

Nusser, Tanja. „Realismus beginnt eigentlich immer, und das von allen Seiten, er ist eine permanente Aufforderung. Über Kathrin Rögglas Texte“. Neue Realismen in der Gegenwartliteratur. Hg. Sǿren R. Fauth und Rolf Parr. Paderborn: Fink, 2016. 213-225.

Ostrowicz, Philipp Alexander. Die Poetik des Möglichen. Das Verhältnis von ,historischer Realität' und, literarischer Wirklichkeit' in Marcel Beyers Roman ,Flughunde‘. Stuttgart: ibidem, 2003.

Picandet, Katharina. Zitatromane der Gegenwart. Georg Schmid ,Roman trouvé‘ - Marcel Beyer ,Das Menschenfleisch“- Thomas Meinecke,Hellblau'. Frankfurt/M.: Peter Lang, 2011.

Pörksen, Bernhard. „Das Problem der Grenze“. Grenzgänger: Formen des New Journalism. Hg. Bernhard Pörksen und Joan Kristin Bleicher. Wiesbaden: VS Verlag für Sozialwissenschaften, 2004. 15-28.

Pörksen, Bernhard, und Joan Kristin Bleicher (Hg.). Grenzgänger: Formen des New Journalism. Wiesbaden: VS Verlag für Sozialwissenschaften, 2004.

Porombka, Stephan. „Really Ground Zero. Die Wiederkehr des Dokumentarischen.“ Literatur der Jahrtausendwende. Themen, Schreibverfahren und Buchmarkt um 2000. Hg. Evi Zemanek und Susanne Kroner. Bielefeld: transcript, 2015. 267-279.

Randers, Jorgen. „2052: Droht ein globaler Kollaps?“. Aus Politik und Zeitgeschichte 62.51-52 (2012): 3-10.

Reinhardt, Michaela. „,Poetische‘ Sprache in zeitgenössischen Theatertexten - am Beispiel von Kathrin Rögglas ,worst case““. Sprache - Literatur - Literatursprache. Hg. Anne Betten und Jürgen Schiewe. Berlin: Erich Schmidt, 2011. 176-196.

Röggla, Kathrin. die alarmbereiten. Frankfurt/M.: Fischer, 2010a.

Röggla, Kathrin. „Beinahe ein Leben auf der Autobahn verbracht“. Manuskripte 36.133 (1996): 110-111.

Röggla, Kathrin. besser wäre: keine. Essays und Theater. Frankfurt/M.: Fischer, 2013.

Röggla, Kathrin. fake reports. Theater, Theater (3). Hg. Uwe B. Carstensen. Frankfurt/M.: Fischer, 2003. 387-434.

Röggla, Kathrin. Die falsche Frage: Theater, Politik und die Kunst, das Fürchten nicht zu verlernen. Berlin: Theater der Zeit, 2014.

Röggla, Kathrin. Irres Wetter. Frankfurt/M.: Fischer, 2002 [2000].

Röggla, Kathrin. Machthaber. kolik 48 (2010b): 27-49.

Röggla, Kathrin. Die Nachtsendung. Unheimliche Geschichten. Frankfurt/M.: Fischer, 2016.

Röggla, Kathrin. really ground zero. Frankfurt/M.: Fischer, 2001. 
Röggla, Kathrin. das stottern des realismus, fiktion und fingiertes, ironie und kritik. Paderborn: Präsidium der Universität, 2011.

Röggla, Kathrin. „Suchbilder. Über Strategien eines literarischen Schreibens, das durch die Katastrophen-Grammatik und Facebook-Talk verzerrte Realität wieder zu fassen bekommt“. TdZ (März 2015): 32-34.

Röggla, Kathrin. „Die Verwörterung der Welt“. Literaturen 5 (2005): 78-83.

Röggla, Kathrin. worst case [Spielfassung]. Frankfurt/M.: Fischer, 2008.

Roß, Dieter. „Fakten und/oder Fiktionen“. Grenzgänger: Formen des New Journalism. Hg. Bernhard Pörksen und Joan Kristin Bleicher. Wiesbaden: VS Verlag für Sozialwissenschaften, 2004. 74-99.

Rueckert, William. „Literature and Ecology: An Experiment in Ecocriticism.“ Iowa Review 9.1 (1978): 71-86.

Rutka, Anna. „Die moderne Katastrophenlust und ihre Narrative. Zu Kathrin Rögglas disaster awareness fair (2006) und die alarmbereiten (2010)“. Genuss und Qual. Hg. Grzegorz Jaśkiewicz und Jan Wolski. Bd. II. Rzeszów: Wydawnictwo Uniwersytetu Rzeszowskiego, 2014, 256-268.

Rutka, Anna. „Zeitgenössische Gesellschaft und ihre Ängste. Zur sprachlichen Re-Inszenierung des Katastrophischen in Kathrin Rögglas Prosaband die alarmbereiten". Germanica Wratislaviensia 139/2014: 99-113.

Schätzing, Frank. Der Schwarm. Köln: Kiepenheuer und Witsch, 2004.

Schaumann, Caroline und Heather I. Sullivan (Hg.). German Ecocriticism in the Anthropocene. New York: Palgrave Macmillan US, 2017.

Schmitt, Claudia und Christiane Solte-Gresser (Hg.). Literatur und Ökologie. Neue literatur- und kulturwissenschaftliche Perspektiven. Bielefeld: transcript, 2017.

Schossböck, Judith. „Letzte Menschen. Die Heldinnen und Helden des Weltuntergangs“. Aus Politik und Zeitgeschichte 62.51-52 (2012): 38-44.

Sonntag, Karlheinz. Von Lissabon bis Fukushima - Folgen von Katastrophen. Heidelberg: Winter, 2013.

Sontag, Susan. Against Interpretation and Other Essays. New York: Picador, 1966.

Sontag, Susan. „Die Katastrophenlust“ (1965). Kunst und Antikunst. 24 literarische Analysen. München und Wien: Hanser, 2003a. 279-300.

Sontag, Susan. Das Leiden anderer betrachten. München und Wien: Hanser, 2003b.

Sontag, Susan. Regarding the Pain of Others. New York: Straus and Giroux, 2003c.

Sontag, Susan. Über Fotografie. München und Wien: Hanser, 2002.

Spengler, Oswald. Der Untergang des Abendlandes. Bd. I. Gestalt und Wirklichkeit. Wien und Leipzig: Wilhelm Braunmüller, 1918.

Spengler, Oswald. Der Untergang des Abendlandes. Bd. II. Welthistorische Perspektiven. München: C. H. Beck, 1922.

Starre, Alexander. „Always already green. Zur Entwicklung und den literaturtheoretischen Prämissen des amerikanischen Ecocriticism“. Ökologische Transformationen und literarische Repräsentationen. Hg. Maren Ermisch, Ulrike Kruse und Urte Stobbe. Göttingen: Universitätsverlag, 2010.13-34.

Steffen, W., Paul J. Crutzen und J. R. McNeill. „The Anthropocene: Are Humans Now Overwhelming the Great Forces of Nature?"Ambio 36 (2007): 614-621.

Stuhlfauth-Trabert, Mara. Seit Jahrzehnten „fünf nach zwölf“. Ökologisches Bewusstsein in Werken von Günter Grass, Andreas Maier, Christine Büchner, Kathrin Röggla und Ilija Trojanow. Würzburg: Ergon, 2017. 
Szczepaniak, Monika. „Elfriede Jelinek und Kathrin Röggla ,in Mediengewittern““. Neue Stimmen aus Österreich. 11 Einblicke in die Literatur der Jahrtausendwende. Hg. Joanna Drynda und Marta Wimmer. Frankfurt/M.: Peter Lang, 2013. 25-35.

Trempler, Jörg. Katastrophen. Ihre Entstehung aus dem Bild. Berlin: Klaus Wagenbach, 2013. Trojanow, Ilja. EisTau. München: dtv, 2011.

Uekötter, Frank. Am Ende der Gewissheiten. Die ökologische Frage im 21. Jahrhundert. Köln: Campus, 2011.

Utz, Peter. Kultivierung der Katastrophe. Literarische Untergangsszenarien aus der Schweiz. München: Wilhelm Fink, 2013.

Uvanović, Željko. „Marshall McLuhan’s Ambivalent Prophecies of Digital Age and Kathrin Röggla’s Pessimistic Diagnoses“. Primerjalna književnost 40.1 (2017): 95-111.

Voltaire. „Gedicht über die Katastrophe von Lissabon“ („Poème sur le désastre de Lisbonne“). Die Erschütterung der vollkommenen Welt. Die Wirkung des Erdbebens von Lissabon im Spiegel europäischer Zeitgenossen. Hg. Wolfgang Breidert. Wissenschaftliche Buchgesellschaft: Darmstadt 1994. 58-76.

Voltaire. Kandide. München: Müller 1912.

Voss, Martin. „Von der Naturkatastrophenforschung zur Suche nach der resilienten Gesellschaft“. Symbolische Formen. Grundlagen und Elemente einer Soziologie der Katastrophe. Hg. Martin Voss. Bielefeld: transcript, 2006. 50-58.

Walter, François. Katastrophen. Eine Kulturgeschichte vom 16. bis ins 21. Jahrhundert. Stuttgart: Reclam, 2010.

Warenski, Brigitte. „Die Angst, die uns Lust verschafft. Kathrin Röggla ist zu Gast bei den Internationalen Literaturtagen ,Sprachsalz‘ in Hall“. Tiroler Tageszeitung (216), 16.09.2006: 16 .

Wellersdorf, Dieter. Der Roman und die Erfahrbarkeit der Welt. Köln: Kiepenheuer \& Witsch, 1988.

Werber, Niels. „Factual Fiction. Zur Differenzierungsgeschichte von Literatur und Journalismus“. Grenzgänger. Formen des New Journalism. Hg. Joan Bleicher und Bernhard Pörksen. Wiesbaden: Verlag für Sozialwissenschaften, 2004. 160-189.

Wilke, Sabine. „Figurationen von Klimawandel: Ilija Trojanows ,EisTau“ als Elegie des Anthropozäns“. Gegenwartsliteratur. Ein germanistisches Jahrbuch / A German Studies Yearbook 13 (2014): 255-274.

Wilde, Sebastian. „Die Wirklichkeit der Katastrophe. Zur medienkritischen Reflexion der Elbwasserdarstellungen 2002 in Marcel Beyers Essay ,Wasserstandsbericht““. Katastrophen. Konfrontationen mit dem Realen. Hg. Solvejg Nitzke und Mark Schmitt. Essen: Christian A. Bachmann, 2012. 129-148.

Wojno-Owczarska, Ewa. „11 września 2001 roku oraz 11 marca 2004 roku w utworach ,really ground zero' Kathrin Röggli i ,Madryt, 11 marca“ Zuzanny Jakubowskiej“. Studia Niemcoznawcze / Studien zur Deutschkunde XLIX (2012): 433-445.

Wojno-Owczarska, Ewa. „Beziehungen zwischen Literatur und Film am Beispiel des Schaffens von Kathrin Röggla“. Zeitschrift des Verbandes Polnischer Germanisten II.4 (2013): 349-368.

Wojno-Owczarska, Ewa. „Essay und Essayismus bei Robert Musil und Kathrin Röggla Versuch eines Vergleichs“. Essay und Essayismus. Hg. Sławomir Leśniak. Gdańsk: Studia Germanica Gedanensia 32 (2015): 47-63.

Wojno-Owczarska, Ewa. „Global Crises in Ernst-Wilhelm Händler’s ,Wenn wir sterben“ and Kathrin Röggla’s ,wir schlafen nicht““. Global Crises and Twenty-First-Century World 
Literature. Hg. Hansong Dan und Ewa Wojno-Owczarska. Comparative Literature Studies [Special Issue] 55.2 (2018): 303-325.

Wojno-Owczarska, Ewa. „Mediale Bilder und filmische Narration bei Kathrin Röggla“. Gegenwartsliteratur. Ein germanistisches Jahrbuch. / A German Yearbook 16 (2017): 257-281.

Wojno-Owczarska, Ewa. „Die österreichische Autorin Kathrin Röggla nimmt den Abstieg des Mittelstandes ins Visier und reflektiert über Tendenzen des Gegenwartstheaters“. http:// www.theaterforschung.de/rezension.php4?ID=2155\&PHPSESSID $=$ c070012800b1960da07 d97b455f4b107 (1. Juni 2016).

Wojno-Owczarska, Ewa. „Der Typus der modernen Frau in Kathrin Rögglas ,wir schlafen nicht““. Studia Niemcoznawcze / Studien zur Deutschkunde 33 (2006): 351-360.

Wojno-Owczarska, Ewa. „Zum Bild von Berlin im Schaffen von Kathrin Röggla“. Acta Philologica 43 (2013): 157-165.

Wojno-Owczarska, Ewa. „Zur Darstellung der Tschernobyl-Katastrophe in Christa Wolfs Störfall. Nachrichten eines Tages und Swetlana Alexijewitschs Tschernobyl. Eine Chronik der Zukunft“. Dialog kultur / Dialog der Kulturen. Hg. Anna Warakomska [u. a.]. Warszawa: Instytut Germanistyki Uniwersytetu Warszawskiego, 2016. 563-585.

Wuketis, Franz M. „Apokalyptische Rhetorik als politisches Druckmittel“. Aus Politik und Zeitgeschichte 62.51-52 (2012): 11-16.

Zapf, Hubert. Literatur als kulturelle Ökologie. Zur kulturellen Funktion imaginativer Texte an Beispielen des amerikanischen Romans. Tübingen: Niemeyer, 2002.

Zemanek, Evi. „Die Kunst der Ökotopie. Zur Ästhetik des Genres und der fiktionsinternen Funktion der Künste (Morus, Morris, Callenbach)“. Ökologie und die Künste. Hg. Erika Fischer-Lichte und Daniela Hahn. München: Wilhelm Fink, 2015. 257-274.

Zemanek, Evi (Hg.). Ökologische Genres. Naturästhetik - Umweltethik - Wissenspoetik. Göttingen: Vandenhoeck \& Ruprecht, 2017.

Zemanek, Evi. „Unkalkulierbare Risiken und ihre Nebenwirkungen. Zu literarischen Reaktionen auf ökologische Transformationen und den Chancen des Ecocriticism“. Literatur als Wagnis/Literature as Risk. Hg. Monika Schmitz-Emans et al. Berlin und Boston: Walter de Gruyter 2013, 279-302.

Ewa Wojno-Owczarska studierte von 1994 bis 1999 Germanistik an der Universität Warschau. Von 1997 bis 1998 war sie zudem DAAD-Stipendiatin an der Friedrich-Schiller-Universität Jena. 1999 schloss sie das Hochschulstudium mit Auszeichnung ab und begann ihr Doktorandenstudium. 2001 erhielt sie ein Stipendium des Bayerischen Staatsministeriums für Forschung, Wissenschaft und Kunst an der Ludwig-Maximilians-Universität München, um ihre Dissertation über die Entwicklung der deutschen Literaturoper im 20. Jahrhundert abzuschließen. Nach der Promotion (2003) begann sie, Material zur Werkmonographie von Kathrin Röggla zusammenzustellen. Seit 2003 arbeitet sie als wissenschaftliche Mitarbeiterin am Germanistischen Institut der Universität Warschau. 
Von 2014 bis 2016 hatte sie ein Stipendium der Alexander von Humboldt-Stiftung für erfahrene Wissenschaftlerinnen zur Vorbereitung der Werkmonographie von Kathrin Röggla am Institut für deutsche Literatur der Humboldt-Universität zu Berlin (Betreuerin an der HU Berlin: Frau Prof. Dr. Ulrike Vedder). Zudem veranstaltete sie drei internationale Konferenzen mit Lesungen von Kathrin Röggla: den internationalen Workshop Literarische Katastrophendiskurse im 20. und 21. Jahrhundert an der HU Berlin (zusammen mit Prof. Dr. Ulrike Vedder, 2015), die internationale Tagung zum Begriff „Arbeit“ im Berliner Brecht-Haus (2016) und die internationale Tagung Topographien der Globalisierung/Topographies of Globalization an der Universität Warschau (2018). Seit 2016 ist sie Leiterin des internationalen Projekts Globalisierungsdiskurse in Literatur und Film des 20. und 21. Jahrhunderts (in Kooperation mit der HU Berlin und der ELTE Budapest). Sie ist Herausgeberin bzw. Mitherausgeberin von 5 Sammelbänden bei Peter Lang und Penn State University Press (Reihe: Comparative Literature Studies). Zudem liegen zahlreiche Publikationen in deutscher, polnischer und englischer Sprache vor. Die von ihr herausgegebene Publikation Topographien der Globalisierung. Norbert Honsza zum Gedächtnis ist zum Zeitpunkt des Erscheinens des vorliegenden Bandes in Vorbereitung. 